\title{
NONLINEAR EARTH ORBIT CONTROL USING LOW-THRUST PROPULSION
}

\author{
Mauro Pontani ${ }^{\star}$ and Marco Pustorino ${ }^{\dagger}$
}

\begin{abstract}
This research is focused on the definition, analysis, and numerical testing of an effective nonlinear orbit control technique tailored to compensating orbit perturbations, as well as possible errors at orbit injection of low- and medium-altitude Earth-orbit satellites. A general, systematic approach to real-time orbit control is presented, under the assumption that the satellite of interest is equipped with a steerable and throttleable low-thrust propulsion system. Two different operational orbits are considered: (a) very-low-altitude Earth orbit and (b) mediumaltitude Earth orbit. A feedback control law based on Lyapunov stability theory is proposed and tested. Some remarkable stability properties are established analytically. Then, the overall performance of the nonlinear control at hand is investigated for cases (a) and (b), over 5 years. The effect of satellite eclipsing on available electrical power is considered as well. For mission scenario (a), suitable tolerances on the desired (nominal) conditions allow substantial savings in terms of propellant requirements.
\end{abstract}

\section{INTRODUCTION}

During their lifetime, orbiting satellites often perform corrective maneuvers, for the purpose of avoiding excessive performance degradation, related to perturbations inherent to the space environment, namely (i) aerodynamic drag, (ii) solar radiation pressure, (iii) Earth gravitational harmonics, and (iv) pull of Sun and Moon as third bodies. The definition and implementation of an effective orbit control strategy thus represents a crucial issue, in order to compensate these perturbation actions, as well as possible errors at orbit injection.

Orbit control regards a variety of satellites, e.g. those that travel low-altitude or geostationary orbits. Well consolidated techniques exist for impulsive orbit control performed through chemical propulsion [1]. These methodologies are based on several fundamental results on optimal impulsive orbit transfers, derived since the 50s [2-11]. An example is represented by the station keeping maneuvers performed by geostationary satellites to compensate the effects of the orbital perturbations, specifically the third body pull due to Sun and Moon and the sectorial harmonic $J_{2,2}$ of the geopotential.

\footnotetext{
* Associate Professor, Department of Astronautical, Electrical, and Energy Engineering, Sapienza University of Rome, via Salaria 851, 00138 Rome, Italy.

$\dagger$ M.S. Student, Faculty of Civil and Industrial Engineering, Sapienza University of Rome, via Eudossiana 16, 00186 Rome, Italy.
} 
In recent years, low-thrust electric propulsion [12] is gaining an increasing relevance and already found application in a variety of mission scenarios, e.g. the NASA Deep Space 1 and the ESA Smart-1 missions [13,14]. Pioneering studies on low-thrust trajectories are due to Edelbaum [15], who was apparently the first scientist to point out the advantages of using low-thrust propulsion in space missions. Most recently, extensive research on the same subject was performed by Petropoulos [16,17], Betts [18-20], Ross [21], and Kechichian [22-25], to name a few. Low-thrust trajectory optimization problems are solved through the use of direct, indirect, or heuristic approaches, sometimes combined in hybrid forms [26,27]. With this regard, Betts [28], Rao [29], and Conway [30] offer excellent overviews of the available methods in spacecraft trajectory optimization. In all the cases of practical interest, getting an optimal solution is a time-demanding task and is completed offline. However, in actual mission scenarios the real-time requirements of compensating the orbit perturbations or correcting the injection conditions often arise. Low-thrust propulsion can represent a valuable option to do this. For the purpose of investigating real-time low-thrust orbit maneuvering, in the scientific literature some authors [31,32] employed the variational equations (written in the Gauss form). Schaub and Alfriend [33] used a combination of Cartesian coordinates and orbit elements in the dynamical framework of formation flying. Neighboring optimal control [34,35] represents an alternative option, with the remarkable feature of minimizing the additional propellant amount needed for correction of the nonnominal flight conditions. However, a neighboring optimal guidance technique requires an optimal reference path, together with all the related state and costate variables, in order to be appliable and effective. In some dynamical frameworks, such as precise orbit maintenance in the presence of perturbations or unmodeled dynamics, identifying an optimal reference trajectory may be very challenging or even impractical.

This study is focused on the definition, analysis, and numerical testing of an orbit control strategy tailored to compensating orbit perturbations and errors at orbit injection in low- and medium-altitude Earth orbits, for satellites equipped with a steerable and throttleable low-thrust ion propulsion system (with an upper bound on the thrust magnitude). Nonsingular equinoctial elements are used for modeling the spacecraft orbit dynamics. The objective consists in obtaining stable flight conditions as close as possible to the desired operational orbit. Lyapunov stability theory and the LaSalle's invariance principle [36-39] are employed with the intent of investigating the convergence properties of a feedback law tailored to low-thrust orbit control. The effect of satellite eclipsing on availability of the onboard electric power is being addressed as well. The nonlinear control technique at hand requires no reference path to be applied, unlike what occurs for neighboring optimal guidance algorithms. Two mission scenarios of practical relevance, corresponding to two distinct operational orbits, are considered: (a) circular, very low Earth orbit and (b) near-circular, medium-altitude Earth orbit. The main objectives of the present research are the following: (i) propose an effective real-time feedback law that includes control saturation, related to the maximum available thrust magnitude, (ii) extend the range of application of nonlinear orbit control through the use of nonsingular equinoctial elements and a rather general formulation for the constraints that define the target orbit, (iii) provide the stability analysis in the presence of perturbing accelerations inherent to the space environment, and (iv) test the orbit control law in the two mentioned mission scenarios, in order to ascertain its effectiveness and efficiency in terms of propellant consumption. 


\section{SPACECRAFT DYNAMICS}

This work employs nonlinear orbit control using low-thrust propulsion, for the purpose of driving and maintaining the spacecraft in the proximity of some desired operational conditions, which can be described in terms of osculating orbit elements. The space vehicle is modeled as a point mass, and its orbital motion around the Earth is subject to some perturbations, described in the next section. Steerable, throttleable low-thrust propulsion actuates the correction maneuvers.

The spacecraft dynamics can be described in terms of either spherical coordinates or osculating orbit elements, i.e. semimajor axis $a$, eccentricity $e$, inclination $i$, right ascension of the ascending node (RAAN) $\Omega$, argument of periapse $\omega$, and true anomaly $f$ [40]. However, the Gauss equations [40], which govern the time evolution of the orbit elements, become singular in the presence of a circular or equatorial orbit (and also when an elliptic orbit transitions to a hyperbola). For these reasons, the nonsingular equinoctial orbit elements [7] $l, m, n, s$, and $q$ are chosen, in conjunction with the semilatus rectum (parameter) $p$, used in place of $a$. The five elements $l, m$, $n, s$, and $q$ are defined as $[7,41,42]$

$$
l=e \cos (\Omega+\omega) \quad m=e \sin (\Omega+\omega) \quad n=\tan \frac{i}{2} \cos \Omega \quad s=\tan \frac{i}{2} \sin \Omega \quad q=\Omega+\omega+f
$$

These elements are nonsingular for all Keplerian trajectories, with the only exception of equatorial retrograde orbits $(i=\pi)$. If $\eta:=1+l \cos q+m \sin q$, the instantaneous radius is $r=p / \eta$. Letting $x_{6} \equiv q$ and $z:=\left[\begin{array}{lllll}x_{1} & x_{2} & x_{3} & x_{4} & x_{5}\end{array}\right]^{T} \equiv\left[\begin{array}{lllll}p & l & m & n & s\end{array}\right]^{T}$, the governing equations for the nonsingular equinoctial elements can be written as

$$
\begin{gathered}
\dot{z}=\mathbf{G}\left(z, x_{6}\right) \boldsymbol{a} \\
\dot{x}_{6}=\sqrt{\frac{\mu_{E}}{x_{1}^{3}}}\left(1+x_{2} \cos x_{6}+x_{3} \sin x_{6}\right)^{2}+\sqrt{\frac{x_{1}}{\mu_{E}}} \frac{x_{4} \sin x_{6}-x_{5} \cos x_{6}}{1+x_{2} \cos x_{6}+x_{3} \sin x_{6}} a_{h}
\end{gathered}
$$

where $\mu_{E}$ represents the Earth gravitational parameter, whereas

$$
\mathbf{G}\left(z, x_{6}\right)=\sqrt{\frac{x_{1}}{\mu_{E}}}\left[\begin{array}{ccc}
0 & \frac{2 x_{1}}{\eta} & 0 \\
\sin x_{6} & \frac{(\eta+1) \cos x_{6}+x_{2}}{\eta} & -\frac{x_{4} \sin x_{6}-x_{5} \cos x_{6}}{\eta} x_{3} \\
-\cos x_{6} & \frac{(\eta+1) \sin x_{6}+x_{3}}{\eta} & \frac{x_{4} \sin x_{6}-x_{5} \cos x_{6}}{\eta} x_{2} \\
0 & 0 & \frac{1+x_{4}^{2}+x_{5}^{2}}{2 \eta} \cos x_{6} \\
0 & 0 & \frac{1+x_{4}^{2}+x_{5}^{2}}{2 \eta} \sin x_{6}
\end{array}\right]
$$

Vector $\boldsymbol{a}$ represents the $(3 \times 1)$-vector of the non-Keplerian acceleration that affects the spacecraft motion. Its components, denoted with $\left(a_{r}, a_{\theta}, a_{h}\right)$, are the projections of $\boldsymbol{a}$ into the local vertical local horizontal (LVLH) rotating frame aligned with $(\hat{r}, \hat{\theta}, \hat{h})$, where unit vector $\hat{r}$ is directed 
toward the instantaneous position vector $\boldsymbol{r}$ (taken from the Earth center), whereas $\hat{h}$ is aligned with the spacecraft angular momentum. Vector $\boldsymbol{a}$ includes both the thrust acceleration and the perturbing acceleration inherent to the space environment. It is convenient to distinguish these two contributions, therefore $\boldsymbol{a}=\boldsymbol{a}_{T}+\boldsymbol{a}_{P}$, where subscripts T and P refer respectively to thrust and perturbations.

Let $T_{\max }$ and $m_{0}$ represent the maximum available thrust magnitude and the initial mass. If $x_{7}$ denotes the mass ratio and $T$ the thrust magnitude, for $x_{7}$ the following equation can be obtained:

$$
\dot{x}_{7}:=\frac{\dot{m}}{m_{0}}=-\frac{u_{T}}{c} \quad \text { with } \quad 0 \leq u_{T} \leq u_{T}^{(\max )} \quad\left(u_{T}:=\frac{T}{m_{0}} \quad \text { and } \quad u_{T}^{(\max )}:=\frac{T_{\max }}{m_{0}}\right)
$$

where $c$ represents the (constant) effective exhaust velocity of the propulsion system. The magnitude of the instantaneous thrust acceleration is $a_{T}=u_{T} m_{0} / m=u_{T} / x_{7}$ and is constrained to the interval $0 \leq a_{T} \leq a_{T}^{(\max )}$, where $a_{T}^{(\max )}=u_{T}^{(\max )} / x_{7}$. Moreover, the thrust acceleration can be expressed as $\boldsymbol{a}_{T}=\boldsymbol{u}_{T} / x_{7}$, where $\boldsymbol{u}_{T}$ has magnitude constrained to the interval $\left[0, u_{T}^{(\max )}\right]$.

In conclusion, the spacecraft dynamics is described in terms of the state vector $\boldsymbol{x}:=\left[\begin{array}{lll}\boldsymbol{z}^{T} & x_{6} & x_{7}\end{array}\right]^{T}=\left[\begin{array}{lllllll}x_{1} & x_{2} & x_{3} & x_{4} & x_{5} & x_{6} & x_{7}\end{array}\right]^{T}$, whereas the control vector is $\boldsymbol{u}_{T}$, directly related to the thrust acceleration. Equations (2), (3), and (5) represent the governing equations.

\section{ORBIT PERTURBATIONS}

While orbiting the Earth, the space vehicle is affected mainly by its gravitational field, and its orbital motion can be investigated appropriately by employing a perturbed two-body-problem model. As a first perturbing action, the Earth gravitational potential differs to some extent from that generated by a spherical mass distribution. As a result, some significant harmonics of the Earth gravitational potential are to be included in the dynamical model, in order to yield more realistic results from simulations. Other than Earth asphericity, the third-body perturbation due to the gravitational attraction of the Moon and the Sun represents an additional contribution. Moreover, for spacecraft that orbit the Earth at (relatively) low altitudes, the drag perturbing acceleration plays a role as well. Lastly, also the perturbing effects due to solar radiation pressure are nonnegligible. This section describes and models all these perturbations.

As a preliminary step, the Earth-centered inertial (ECI) frame is introduced. It is associated with the right-hand sequence of unit vectors $\left(\hat{c}_{1}, \hat{c}_{2}, \hat{c}_{3}\right)$, where $\left(\hat{c}_{1}, \hat{c}_{2}\right)$ identifies the equatorial plane, $\hat{c}_{1}$ is the vernal axis, and $\hat{c}_{3}$ is aligned with the Earth rotation axis. Another useful frame (rotating together with the spacecraft) is associated with $(\hat{r}, \hat{E}, \hat{N})$, where $\hat{E}$ and $\hat{N}$ are aligned with the local East and North directions, respectively. Angles $\xi$ (absolute longitude), $\phi$ (latitude), and $\zeta$ (heading) relate the ECI-frame to the $(\hat{r}, \hat{E}, \hat{N})$-frame and to the $(\hat{r}, \hat{\theta}, \hat{h})$-frame,

$$
\left[\begin{array}{lll}
\hat{r} & \hat{\theta} & \hat{h}
\end{array}\right]^{T}=\mathbf{R}_{1}(\zeta)\left[\begin{array}{lll}
\hat{r} & \hat{E} & \hat{N}
\end{array}\right]^{T}=\mathbf{R}_{1}(\zeta) \mathbf{R}_{2}(-\phi) \mathbf{R}_{3}(\xi)\left[\begin{array}{lll}
\hat{c}_{1} & \hat{c}_{2} & \hat{c}_{3}
\end{array}\right]^{T}
$$


where $\mathbf{R}_{j}(\chi)$ denotes the matrix associated with the elementary counterclockwise rotation by angle $\chi$ about axis $j$. Moreover, an additional relation between the ECI-frame and the $(\hat{r}, \hat{\theta}, \hat{h})$ frame can be written in terms of orbit elements [40],

$$
\left[\begin{array}{lll}
\hat{r} & \hat{\theta} & \hat{h}
\end{array}\right]^{T}=\mathbf{R}_{3}(\omega+f) \mathbf{R}_{1}(i) \mathbf{R}_{3}(\Omega)\left[\begin{array}{lll}
\hat{c}_{1} & \hat{c}_{2} & \hat{c}_{3}
\end{array}\right]^{T}
$$

\subsection{Earth gravitational harmonics}

In the last decades, several accurate models have been developed for the Earth gravitational field, e.g. EIGEN05S, GGM03S, and EGM96, to name a few. This research employs the EGM2008 model [43], which supplies the coefficients of zonal, tesseral, and sectorial harmonics of the Earth gravitational field up to order 2160. These coefficients $\left(J_{l, m}\right.$ and $\left.\lambda_{l m}\right)$ appear in the classical equation of planetary gravitational potentials (per mass unit), written in terms of Legendre polynomials $P_{l m}$,

$$
U=\frac{\mu_{E}}{r}-\frac{\mu_{E}}{r} \sum_{l=2}^{\infty}\left(\frac{R_{E}}{r}\right)^{l} J_{l} P_{l 0}(\sin \phi)+\frac{\mu_{E}}{r} \sum_{l=2}^{\infty} \sum_{m=1}^{l}\left(\frac{R_{E}}{r}\right)^{l} J_{l, m} P_{l m}(\sin \phi) \cos \left[m\left(\lambda_{g}-\lambda_{l m}\right)\right]
$$

where $R_{E}$ is the Earth equatorial radius, whereas $\lambda_{g}$ is the spacecraft geographical longitude (taken from the Greenwich reference meridian). If $\theta_{G}$ denotes the Greenwich sidereal time (taken counterclockwise from $\hat{c}_{1}$ ), then the satellite geographical longitude is $\lambda_{g}=\xi-\theta_{G}$. Both the latitude $\phi$ and the absolute longitude $\xi$ can be expressed as functions of the orbit elements by comparing Eqs. (6) and (7). The latter variables in turn can be written in terms of equinoctial elements using Eq. (1).

In the $(\hat{r}, \hat{E}, \hat{N})$-frame, the gravitational acceleration is given by

$$
\boldsymbol{g}=\nabla U \quad \text { where } \nabla=\hat{r} \frac{\partial}{\partial r}+\frac{\hat{E}}{r \cos \phi} \frac{\partial}{\partial \lambda_{g}}+\frac{\hat{N}}{r} \frac{\partial}{\partial \phi}
$$

The previous expression, together with Eq. (8), leads to obtaining the three components $\left(g_{r}, g_{E}, g_{N}\right)$ in the local horizontal $(\hat{r}, \hat{E}, \hat{N})$-frame. Because $g_{r}$ includes the main gravitational term, the related disturbing acceleration components are $a_{r}^{(H)}=g_{r}+\mu / r^{2}, a_{E}^{(H)}=g_{E}$, and $a_{N}^{(H)}=g_{N}$. Using Eqs. (6)-(7), the components $\left(a_{r}^{(H)}, a_{\theta}^{(H)}, a_{h}^{(H)}\right)$ of $\boldsymbol{a}^{(H)}$ along $(\hat{r}, \hat{\theta}, \hat{h})$ can be obtained in a straightforward way. In this study, all the harmonics associated with $\left|J_{l, m}\right|>10^{-6}$ are considered, i.e. $J_{2}, J_{3}, J_{4}, J_{2,2}$, and $J_{3,1}$.

\subsection{Third body perturbation}

The Moon and Sun gravitational influence on the space vehicle while this orbits the Earth can be modeled as a third body perturbation. In general, the perturbing acceleration due to a third body can be expressed as 


$$
\boldsymbol{a}^{(3 B)}=-\frac{\mu_{3}}{s_{3}^{3}\left(1+q_{3}\right)^{3 / 2}}\left[\boldsymbol{r}+\boldsymbol{s}_{3} q_{3} \frac{3+3 q_{3}+q_{3}^{2}}{1+\left(1+q_{3}\right)^{3 / 2}}\right], \quad \text { with } \quad q_{3}:=\frac{r^{2}-2 \boldsymbol{r}^{T} \boldsymbol{s}_{3}}{s_{3}^{2}}
$$

where $\mu_{3}$ denotes the gravitational parameter of the third body, $s_{3}$ represents its position vector relative to the main body (i.e., the Earth), and $s_{3}=\left|s_{3}\right|$. The previous expression makes use of the Battin-Giorgi $[7,44]$ approach to the Encke's method for orbit perturbations.

In the ECI-frame, the instantaneous positions of both the Sun and the Moon can be derived through interpolation of the ephemerides, using the approach described in Ref. 45. Then, $\boldsymbol{s}_{3}$ can be projected onto the LVLH-frame using Eq. (7), to yield the three components of the perturbing accelerations, i.e. $\left(a_{r}^{(S)}, a_{\theta}^{(S)}, a_{h}^{(S)}\right)$ for the Sun and $\left(a_{r}^{(M)}, a_{\theta}^{(M)}, a_{h}^{(M)}\right)$ for the Moon. If $\boldsymbol{a}^{(S)}$ and $\boldsymbol{a}^{(M)}$ denote the related vectors, the overall perturbing acceleration due to the gravitational pull of third bodies is $\boldsymbol{a}^{(3 B)}=\boldsymbol{a}^{(S)}+\boldsymbol{a}^{(M)}$.

\subsection{Aerodynamic drag}

If the spacecraft orbits the Earth at relatively low altitudes (lower than $1000 \mathrm{~km}$ ), also the aerodynamic drag is to be considered as a perturbing action. Let $S_{D}$ and $c_{D}$ denote the aerodynamic cross section and drag coefficient of the space vehicle. The drag acceleration $\boldsymbol{a}^{(D)}$ is given by

$$
\boldsymbol{a}^{(D)}=-\frac{1}{2} c_{D} \frac{S_{D}}{m} \rho v_{R} \boldsymbol{v}_{R}
$$

where $\rho$ is the local atmospheric density, $v_{R}$ is the spacecraft velocity relative to the atmosphere, and $v_{R}=\left|v_{R}\right|$. In this research $\rho$ is interpolated by means of a piecewise exponential function, based on tabular data [46]. As orbital motion takes place at hypersonic velocities in rarefied flow regime, $c_{D}$ is nearly constant, thus it is set to the typical value 2.2. Under the assumption that the atmosphere rotates together with the Earth, the relative velocity is

$$
\boldsymbol{v}_{R}=\boldsymbol{v}-\omega_{E} r \cos \phi \hat{E}
$$

where $v$ denotes the spacecraft inertial velocity, given by

$$
\boldsymbol{v}=\sqrt{\frac{\mu_{E}}{p}}[\hat{r} e \sin f+\hat{\theta}(1+e \cos f)]
$$

Moreover, $\hat{E}$ can be written as $\hat{E}=\hat{\theta} \cos \zeta-\hat{h} \sin \zeta$, while both $\phi$ and $\zeta$ can be expressed as a function of the orbit elements by means of Eqs. (6)-(7). These can be written again in terms of equinoctial elements using Eq. (1). As a result, $v_{R}$ can be finally projected in the LVLH-frame, as well as the drag acceleration $\boldsymbol{a}^{(D)}$. The respective components are denoted with $\left(a_{r}^{(D)}, a_{\theta}^{(D)}, a_{h}^{(D)}\right)$.

\subsection{Solar radiation pressure}

Solar radiation pressure is associated with a further perturbing acceleration and derives from the interaction of photons with the spacecraft when this is illuminated. For the sake of simplicity, the cannonball model is adopted [45], and the perturbing acceleration due to solar radiation is 


$$
\boldsymbol{a}^{(S R)}=-v P_{S R} \frac{c_{R} S_{R}}{m} \hat{r}_{S}
$$

where $P_{S R}\left(=4.557 \cdot 10^{-6} \mathrm{~Pa}\right)$ is the solar radiation pressure on Earth, $c_{R}$ is the radiative coefficient, related to the nature of the radiation interaction with the space vehicle, $S_{R}$ is the spacecraft cross section that is illuminated, $\hat{r}_{S}$ is the unit vector aligned with the Sun position vector $\boldsymbol{r}_{S}$ (taken from the Earth center), and $v$ is the shadow function. In this study, $c_{R}$ is set to 2 (perfect reflection). In the ECI-frame, the instantaneous position of the Sun, $\boldsymbol{r}_{S}$, is interpolated using again the approach described in Ref. 45. Then, $\boldsymbol{r}_{S}$ can be projected onto the LVLH-frame using Eq. (7), to yield the three components of the perturbing accelerations, i.e. $\left(a_{r}^{(S R)}, a_{\theta}^{(S R)}, a_{h}^{(S R)}\right)$.

The shadow function $v$ equals either 0 (when the space vehicle is eclipsed) or 1 (when it is illuminated). Letting $\vartheta_{1}:=\arccos \left(R_{E} / r\right), \quad \vartheta_{2}:=\arccos \left(R_{E} / r_{S}\right) \quad$ (with $\quad r_{S}=\left|r_{S}\right|$ ), and $\varphi:=\arccos \left(\hat{r} \cdot \hat{r}_{S}\right)$, the space vehicle is eclipsed if [45]

$$
\varphi>\vartheta_{1}+\vartheta_{2}
$$

Because $R_{E} / r_{S} \approx 0$, the previous relation becomes

$$
\cos \varphi<-\sin \vartheta_{1}=-\sqrt{1-\left(\frac{R_{E}}{r}\right)^{2}}
$$

The term $\cos \varphi$ can be computed easily, after writing $\hat{r}$ and $\hat{r}_{S}$ in the ECI-frame. Therefore, if inequality (16) is satisfied, then $v=0$, otherwise $v=1$.

\section{NONLINEAR ORBIT CONTROL: DEFINITION AND STABILITY ANALYSIS}

Previous research [32] has shown that any state (associated with elliptic orbits) is accessible when the spacecraft dynamics is subject to the Gauss equations for classical orbit elements. However, the same property holds also for equinoctial elements [32]. This represents the theoretical premise for applying nonlinear techniques to orbital control.

In a preceding section, the spacecraft motion was shown to be governed by Eqs. (2), (3), and (5). In particular, Eq. (2) can be rewritten as

$$
\dot{z}=\mathbf{G}\left(z, x_{6}\right)\left(\frac{\boldsymbol{u}_{T}}{x_{7}}+a_{P}\right)
$$

where the perturbing acceleration $\boldsymbol{a}_{P}$ includes several contributions, related to the space environment, namely harmonics of the geopotential (term $\boldsymbol{a}^{(H)}$ ), third body gravitational attraction $\left(\boldsymbol{a}^{(3 B)}\right)$, aerodynamic drag $\left(\boldsymbol{a}^{(D)}\right)$, and solar radiation pressure $\left(\boldsymbol{a}^{(S R)}\right)$. Thus, $\boldsymbol{a}_{P}=\boldsymbol{a}^{(H)}+\boldsymbol{a}^{(3 B)}+\boldsymbol{a}^{(D)}+\boldsymbol{a}^{(S R)}$. It is worth noticing that Eq. (17) assumes a control-affine form in the absence of perturbing accelerations $\left(\boldsymbol{a}_{P}=\mathbf{0}\right)$. For systems governed by Eq. (17) with $\boldsymbol{a}_{P}=\mathbf{0}$, the Jurdjevic-Quinn theorem [47] provides a feedback control law that can drive the dynamical system to an arbitrary target state, making the controlled system Lyapunov-stable. 
In practical mission scenarios, orbit maintenance regards some (or all) of the following orbit elements: semimajor axis $a$, eccentricity $e$, inclination $i$, right ascension of the ascending node (RAAN) $\Omega$, and argument of periapse $\omega$. These identify the orbit size, shape, and orientation in space. Under the assumption that the target trajectory is defined in terms of these orbit elements only, the desired operational conditions depend only on $z$ (cf. Eq. (1)) and can be formally defined by

$$
\psi(z)=\mathbf{0}
$$

The previous vector equation is problem-dependent and corresponds to $q(\leq 5)$ relations that involve equinoctial elements $x_{1}$ through $x_{5}$. If $q<5$, Eq. (18) identifies a target set that is assumed to be a connected and differentiable manifold.

\subsection{Lyapunov stability}

This section is specifically devoted to defining a feedback control law capable of driving the dynamical system at hand (associated with Eqs. (3), (5), and (17)) toward the target conditions identified by Eq. (18). To do this, the following candidate Lyapunov function is introduced:

$$
V=\frac{1}{2} \psi^{T} \mathbf{K} \psi
$$

where $\mathbf{K}$ denotes a diagonal matrix with constant, positive elements, which play the role of arbitrary weights. These are selected a priori in relation to the application of interest. It is immediate to recognize that $V>0$ unless $\boldsymbol{\psi}=\mathbf{0}$. Yet, further conditions are required in order that $V$ be an actual Lyapunov function. This issue is being addressed in the following.

Proposition 1. Let $\boldsymbol{b}:=\mathbf{G}^{T}(\partial \boldsymbol{\psi} / \partial \boldsymbol{z})^{T} \mathbf{K} \boldsymbol{\psi}$. If and $\boldsymbol{\psi}$ and $(\partial \boldsymbol{\psi} / \partial \boldsymbol{z})$ are continuous, $|\boldsymbol{b}|>0$ unless $\boldsymbol{\psi}=\mathbf{0}$, and $\boldsymbol{u}_{T}^{(\max )} \geq x_{7}\left|\boldsymbol{b}+\boldsymbol{a}_{P}\right|$, then the feedback control law

$$
\boldsymbol{u}_{T}=-x_{7}\left(\boldsymbol{b}+\boldsymbol{a}_{P}\right)
$$

leads a dynamical system governed by Eqs. (3), (5), and (17) to converge asymptotically to the target set associated with Eq. (18).

Proof. The candidate function (19) can be proven to be an actual Lyapunov function. First, it is apparent that $V$ is positive in the entire domain, except when $\boldsymbol{\psi}=\mathbf{0}$. Second, $V$ has continuous partial derivatives, because $\psi$ and $(\partial \boldsymbol{\psi} / \partial \boldsymbol{z})$ are continuous. Moreover, using Eq. (17), the time derivative of $V$ equals

$$
\dot{V}=\boldsymbol{\psi}^{T}(\boldsymbol{z}) \mathbf{K}\left(\frac{\partial \boldsymbol{\psi}}{\partial \boldsymbol{z}}\right) \dot{\boldsymbol{z}}=\boldsymbol{\psi}^{T}(\boldsymbol{z}) \mathbf{K}\left(\frac{\partial \boldsymbol{\psi}}{\partial \boldsymbol{z}}\right) \mathbf{G}\left(\frac{\boldsymbol{u}_{T}}{x_{7}}+\boldsymbol{a}_{P}\right)
$$

Insertion of Eq. (20) yields

$$
\dot{V}=-\boldsymbol{\psi}^{T}(\boldsymbol{z}) \mathbf{K}\left(\frac{\partial \boldsymbol{\psi}}{\partial \boldsymbol{z}}\right) \mathbf{G} \boldsymbol{b}=-\boldsymbol{b}^{T} \boldsymbol{b}<0
$$

which is continuous and negative if $\boldsymbol{\psi} \neq \mathbf{0}$. Definitely, $V$ is a positive definite function (about $\boldsymbol{\psi}=\mathbf{0}$ ), with continuous partial derivatives and such that $\dot{V}<0$ (unless $\boldsymbol{\psi}=\mathbf{0}$ ), therefore $V$ is a 
Lyapunov function [36]. Attraction toward the set $\boldsymbol{\psi}=\mathbf{0}$ implies asymptotic convergence of the dynamical system toward the final condition (18).

The previous proposition includes the assumption $u_{T}^{(\max )} \geq x_{7}\left|\boldsymbol{b}+\boldsymbol{a}_{P}\right|$. If this condition is violated, the feedback control law (20) is infeasible, because $\left|\boldsymbol{u}_{T}\right|=x_{7}\left|\boldsymbol{b}+\boldsymbol{a}_{P}\right|$ would exceed the maximal value $u_{T}^{(\max )}$. In this case, in place of (20), an alternative feedback law can be used.

Proposition 2. Let $\boldsymbol{b}:=\mathbf{G}^{T}(\partial \boldsymbol{\psi} / \partial \boldsymbol{z})^{T} \mathbf{K} \psi$. If and $\boldsymbol{\psi}$ and $(\partial \boldsymbol{\psi} / \partial \boldsymbol{z})$ are continuous, $|\boldsymbol{b}|>0$ unless $\boldsymbol{\psi}=\mathbf{0}, u_{T}^{(\max )}<x_{7}\left|\boldsymbol{b}+\boldsymbol{a}_{P}\right|$, and $\boldsymbol{b}^{T} \boldsymbol{a}_{P} \leq 0$, then the feedback control law

$$
\boldsymbol{u}_{T}=-u_{T}^{(\max )} \frac{\boldsymbol{b}+\boldsymbol{a}_{P}}{\left|\boldsymbol{b}+\boldsymbol{a}_{P}\right|}
$$

leads a dynamical system governed by Eqs. (3), (5), and (17) to converge asymptotically to the target set associated with Eq. (18).

Proof. The candidate function (19) can be proven again to be an actual Lyapunov function. First, it is apparent that $V$ is positive in the entire domain, except when $\boldsymbol{\psi}=\mathbf{0}$. Second, $V$ has continuous partial derivatives, because $\psi$ and $(\partial \boldsymbol{\psi} / \partial \boldsymbol{z})$ are continuous. Moreover, the time derivative of $V$ is reported in Eq. (21), and insertion of Eq. (23) yields

$$
\dot{V}=\boldsymbol{\psi}^{T}(\boldsymbol{z}) \mathbf{K}\left(\frac{\partial \boldsymbol{\psi}}{\partial \boldsymbol{z}}\right) \mathbf{G}\left[\boldsymbol{a}_{P}-\frac{u_{T}^{(\max )}}{x_{7}} \frac{\boldsymbol{b}+\boldsymbol{a}_{P}}{\left|\boldsymbol{b}+\boldsymbol{a}_{P}\right|}\right]=-\frac{u_{T}^{(\max )} \boldsymbol{b}^{T} \boldsymbol{b}}{x_{7}\left|\boldsymbol{b}+\boldsymbol{a}_{P}\right|}+\frac{\boldsymbol{b}^{T} \boldsymbol{a}_{P}\left(x_{7}\left|\boldsymbol{b}+\boldsymbol{a}_{P}\right|-u_{T}^{(\max )}\right)}{x_{7}\left|\boldsymbol{b}+\boldsymbol{a}_{P}\right|}
$$

The first term of Eq. (24) is negative (unless $\boldsymbol{\psi}=\mathbf{0}$ ). Instead, the second term can be either positive or negative, depending on $\boldsymbol{b}^{T} \boldsymbol{a}_{P}$, because $x_{7}\left|\boldsymbol{b}+\boldsymbol{a}_{P}\right|>u_{T}^{(\max )}$. If the inequality $\boldsymbol{b}^{T} \boldsymbol{a}_{P} \leq 0$ is fulfilled, then the sum in the right-hand side of Eq. (24) is negative (unless $\boldsymbol{\psi}=\mathbf{0}$ ). Thus, $\dot{V}$ is continuous and negative if the assumptions of the statement hold. Because $V$ is a positive definite function (about $\boldsymbol{\psi}=\mathbf{0}$ ), with continuous partial derivatives and such that $\dot{V}<0$ (unless $\boldsymbol{\psi}=\mathbf{0}$ ), $V$ is a Lyapunov function [36]. Attraction toward the set $\boldsymbol{\psi}=\mathbf{0}$ implies asymptotic convergence of the dynamical system toward the final condition (18).

It is worth remarking that the previous proposition requires the sufficient condition $\boldsymbol{b}^{T} \boldsymbol{a}_{P} \leq 0$ to ensure $\dot{V}<0$. However, the sign of $\boldsymbol{b}^{T} \boldsymbol{a}_{P}$ is time-varying and depends on the particular time evolution of the dynamical system of interest. In fact, $\boldsymbol{b}^{T} \boldsymbol{a}_{P}$ usually assumes both positive and negative values. An additional sufficient condition that ensures $\dot{V}<0$ even if $\boldsymbol{b}^{T} \boldsymbol{a}_{P}>0$, regardless of the specific time evolution, is provided by the following

Proposition 3. Let $\boldsymbol{b}:=\mathbf{G}^{T}(\partial \boldsymbol{\psi} / \partial \boldsymbol{z})^{T} \mathbf{K} \boldsymbol{\psi}$. If and $\boldsymbol{\psi}$ and $(\partial \boldsymbol{\psi} / \partial \boldsymbol{z})$ are continuous, $|\boldsymbol{b}|>0$ unless $\boldsymbol{\psi}=\mathbf{0}$, and $x_{7}\left|\boldsymbol{a}_{P}\right|<u_{T}^{(\max )}<x_{7}\left|\boldsymbol{b}+\boldsymbol{a}_{P}\right|$, then the feedback control law (23) leads a dynamical system governed by Eqs. (3), (5), and (17) to converge asymptotically to the target set associated with Eq. (18).

Proof. The first steps for demonstrating the preceding statement are analogous to those of Propositions 1 and 2. The new condition $u_{T}^{(\max )}>x_{7}\left|\boldsymbol{a}_{P}\right|$ implies $\dot{V}<0$. To prove this, let 
$\chi:=\boldsymbol{b}^{T} \boldsymbol{a}_{P}, a_{P}:=\left|\boldsymbol{a}_{P}\right|$, and $b:=|\boldsymbol{b}|$. Two cases can occur: (i) $\chi \leq 0$ or (ii) $\chi>0$. Case (i) corresponds to $\dot{V}<0$, regardless of $a_{P}$ and $u_{T}^{(\max )}$ (cf. Proposition 2). For case (ii), as a first step, the following inequality is considered:

$$
a_{P} \geq \frac{\chi\left|\boldsymbol{b}+\boldsymbol{a}_{P}\right|}{\boldsymbol{b}^{T}\left(\boldsymbol{b}+\boldsymbol{a}_{P}\right)}
$$

This can be proven by squaring both sides

$$
a_{P}^{2} \geq \frac{\chi^{2}\left(b^{2}+a_{P}^{2}+2 \chi\right)}{\left(b^{2}+\chi\right)^{2}} \Leftrightarrow a_{P}^{2} \geq \frac{\chi^{2}}{b^{2}} \quad \Leftrightarrow \quad a_{P} \geq \frac{\boldsymbol{b}^{T} \boldsymbol{a}_{P}}{b}
$$

If the right-hand side of the last expression is regarded as a dot product, it is straightforward to recognize that all the inequalities in Eqs. (26) hold, and so does Eq. (25). Due to the inequality $u_{T}^{(\max )}>x_{7}\left|\boldsymbol{a}_{P}\right|$ and Eq. (25),

$$
\frac{u_{T}^{(\max )}}{x_{7}}>a_{P} \geq \frac{\chi\left|\boldsymbol{b}+\boldsymbol{a}_{P}\right|}{\boldsymbol{b}^{T}\left(\boldsymbol{b}+\boldsymbol{a}_{P}\right)} \quad \text { i.e. } \quad u_{T}^{(\max )} \boldsymbol{b}^{T}\left(\boldsymbol{b}+\boldsymbol{a}_{P}\right)-x_{7} \boldsymbol{b}^{T} \boldsymbol{a}_{P}\left|\boldsymbol{b}+\boldsymbol{a}_{P}\right|>0
$$

From inspection of Eq. (24), it is apparent that inequality (27) implies $\dot{V}<0$.

The two feedback laws (20) and (23) can be written in compact form as

$$
\boldsymbol{u}_{T}=-u_{T}^{(\max )} \frac{x_{7}\left(\boldsymbol{b}+\boldsymbol{a}_{P}\right)}{\max \left\{u_{T}^{(\max )}, x_{7}\left|\boldsymbol{b}+\boldsymbol{a}_{P}\right|\right\}}
$$

Equation (28) incorporates the saturation condition on $\boldsymbol{u}_{T}$, i.e. $\left|\boldsymbol{u}_{T}\right| \leq u_{T}^{(\max )}$, and provides a control law that can be actuated using steerable and throttleable propulsive thrust (with time-varying magnitude and direction).

Propositions 1 and 2 provide some sufficient conditions for stabilizing the dynamical system of interest. If perturbations are absent $\left(\boldsymbol{a}_{P}=\mathbf{0}\right)$, Eq. (23) provides a Lyapunov-optimal feedback control law [1]. If the perturbing acceleration is negligible with respect to the thrust acceleration $\left(\left|\boldsymbol{a}_{P}\right| \ll u_{T}^{(\max )} / x_{7}\right)$, then the control law (23) can be regarded as nearly-Lyapunov-optimal. Proposition 3 provides a very useful sufficient condition that has a straightforward meaning: if the thrust acceleration magnitude, $u_{T}^{(\max )} / x_{7}$, exceeds the perturbation acceleration magnitude, $a_{P}$, then $\dot{V}<0$ unless $\boldsymbol{\psi}=\mathbf{0}$. As a final remark, it is worth stressing that Propositions 1 through 3 state some sufficient conditions for Lyapunov stability. This circumstance implies that the assumptions of Propositions 1 through 3 can be violated (in some time intervals), without necessarily compromising asymptotic convergence to the desired final condition identified by Eq. (18).

\subsection{Nonlinear control for semimajor axis, eccentricity, and inclination of Earth orbits}

The previous stability properties refer to the spacecraft dynamics, governed by Eqs. (3), (5), and (17). The desired operational conditions are defined by Eq. (18), which is problem-dependent and identifies the target set. 
In this subsection, nonlinear orbit control using low-thrust propulsion is addressed, with the objective of driving the spacecraft toward the desired Earth orbit. This has specified (desired) values of semimajor axis, eccentricity, and inclination, denoted respectively with $a_{d}, e_{d}$, and $i_{d}$. Due to the definitions of Eq. (1), the desired operational conditions correspond to

$$
\begin{gathered}
x_{1}-p_{d}=0 \\
x_{2}^{2}+x_{3}^{2}-e_{d}^{2}=0 \\
x_{4}^{2}+x_{5}^{2}-\tan ^{2} \frac{i_{d}}{2}=0
\end{gathered}
$$

where $p_{d}=a_{d}\left(1-e_{d}^{2}\right)$ and $e_{d}<1$. The left-hand sides of Eqs. (29)-(31) form the vector $\psi$.

The preceding section supplies three sets of sufficient conditions (stated in Propositions 1 through 3) that ensure asymptotic stability, i.e. convergence toward the target set, identified by Eqs. (29)-(31). This subsection is intended to check these conditions, for the $(3 \times 1)$-vector $\psi$ defined by Eqs. (29)-(31).

As first steps, both $\psi$ and $(\partial \psi / \partial z)$ turn out to be continuous in the entire domain where equinoctial elements are defined (i.e., $i \neq \pi$ ).

Then, vector $\boldsymbol{b}$, whose components are $\left\{b_{1}, b_{2}, b_{3}\right\}$, is derived analytically for the problem at hand,

$$
\begin{gathered}
b_{1}=-2 k_{2} \sqrt{\frac{x_{1}}{\mu_{E}}}\left(x_{3} \cos x_{6}-x_{2} \sin x_{6}\right)\left(x_{2}^{2}+x_{3}^{2}-e_{d}^{2}\right) \\
b_{2}=\frac{2}{\eta} \sqrt{\frac{x_{1}}{\mu_{E}}}\left\{k_{1} \sqrt{x_{1}}\left(x_{1}-p_{d}\right)+k_{2}\left(x_{2}^{2}+x_{3}^{2}-e_{d}^{2}\right)\left[\eta^{2}+x_{2}^{2}+x_{3}^{2}-1\right]\right\} \\
b_{3}=\frac{k_{3}}{\eta} \sqrt{\frac{x_{1}}{\mu_{E}}}\left(x_{4} \cos x_{6}+x_{5} \sin x_{6}\right)\left(x_{4}^{2}+x_{5}^{2}-\tan ^{2} \frac{i_{d}}{2}\right)\left(x_{4}^{2}+x_{5}^{2}+1\right)
\end{gathered}
$$

The attracting set collects all the dynamical states that fulfill $\dot{V}=0$. In fact, out of the attracting set $\dot{V}<0$. The latter condition is met if $\boldsymbol{b}=\mathbf{0}$, i.e. if the three components $\left\{b_{1}, b_{2}, b_{3}\right\}$ equal 0 , for any choice of the positive coefficients $\left\{k_{1}, k_{2}, k_{3}\right\}$. It is straightforward to recognize that $x_{1}=0$ yields $b_{1}=b_{2}=b_{3}=0$. Other than this solution, from inspection of Eq. (33), $b_{2}=0$ regardless of $\left\{k_{1}, k_{2}\right\}$ only if $x_{1}=p_{d}$ and $x_{2}^{2}+x_{3}^{2}=e_{d}^{2}$. The alternative condition $\eta^{2}+x_{2}^{2}+x_{3}^{2}-1=0$ is ruled out because $\eta$ depends on $x_{6}$, which is time-varying (also along the desired orbit). Then, $b_{1}=0$ if either $x_{2}^{2}+x_{3}^{2}=e_{d}^{2}$ or $x_{2}=x_{3}=0$. However, the latter condition is ruled out because it would imply $b_{2} \neq 0$. Lastly, $b_{3}=0$ if either $x_{4}^{2}+x_{5}^{2}=\tan ^{2}\left(i_{d} / 2\right)$ or $x_{4}=x_{5}=0$. In short, the attracting set includes the following three subsets: 
1. $x_{1}=0$ (rectilinear trajectories);

2. $x_{1}=p_{d}, x_{2}^{2}+x_{3}^{2}=e_{d}^{2}$, and $x_{4}=x_{5}=0$ (equatorial elliptic orbits with semilatus rectum $p_{d}$ and eccentricity $\left.e_{d}\right)$;

3. $x_{1}=p_{d}, x_{2}^{2}+x_{3}^{2}=e_{d}^{2}$, and $x_{4}^{2}+x_{5}^{2}=\tan ^{2}\left(i_{d} / 2\right)$ (operational conditions, cf. Eqs. (29)$(31))$.

Because the attracting set contains other subsets other than the target set (which coincides with subset 3), the asymptotic convergence toward the desired conditions is only local, based on Lyapunov's stability theorem [38]. However, the LaSalle's principle [38] can be applied in order to rule out, if possible, subsets 1 and 2 . Because $\psi$ is continuous and $\dot{V}<0$ (except in the attracting set, denoted with A henceforth), the condition $V(z) \leq V\left(z_{0}\right)$ (where $z_{0}$ is $z$ evaluated at the initial time) defines a compact set $C$. The invariant set, which plays a crucial role in the LaSalle's principle, is to be sought in $\mathrm{A} \cap \mathrm{C}$, i.e. in the portion of the attracting set contained in $\mathrm{C}$. By definition, the invariant set collects all the dynamical states (in the attracting set of $z$ ) that remain unaltered when $\boldsymbol{a} \equiv \mathbf{0}$. This means that once the invariant set is reached, $\boldsymbol{b} \equiv \mathbf{0}$ at future times, which implies $\dot{\boldsymbol{b}} \equiv \mathbf{0}$ while $\boldsymbol{a} \equiv \mathbf{0}$.

For the application at hand, the time derivatives of the three components of $\boldsymbol{b}$ assume the form

$$
\dot{b}_{j}=\frac{\partial b_{j}}{\partial z} \dot{z}+\frac{\partial b_{j}}{\partial x_{6}} \dot{x}_{6}=\frac{\partial b_{j}}{\partial z} \mathbf{G}\left(z, x_{6}\right) \boldsymbol{a}+\frac{\partial b_{j}}{\partial x_{6}}\left[\sqrt{\frac{\mu_{E}}{x_{1}^{3}}} \eta^{2}+\sqrt{\frac{x_{1}}{\mu_{E}}} \frac{x_{4} \sin x_{6}-x_{5} \cos x_{6}}{\eta} a_{h}\right]
$$

where $j=1,2,3$. The previous expression, evaluated at $\boldsymbol{a} \equiv \mathbf{0}$ reduces to

$$
\dot{b}_{j}=\frac{\partial b_{j}}{\partial x_{6}} \sqrt{\frac{\mu_{E}}{x_{1}^{3}}} \eta^{2} \quad(j=1,2,3)
$$

Using Eqs. (32) through (34) one obtains

$$
\begin{gathered}
\dot{b}_{1}=\frac{2 k_{2}}{x_{1}} \eta^{2}\left(x_{2} \cos x_{6}+x_{3} \sin x_{6}\right)\left(x_{2}^{2}+x_{3}^{2}-e_{d}^{2}\right) \\
\dot{b}_{2}=\frac{2}{x_{1}}\left(x_{2} \sin x_{6}-x_{3} \cos x_{6}\right)\left\{k_{1} \sqrt{x_{1}}\left(x_{1}-p_{d}\right)+k_{2}\left(x_{2}^{2}+x_{3}^{2}-e_{d}^{2}\right)\left[\eta^{2}+x_{2}^{2}+x_{3}^{2}-1\right]\right\} \\
-\frac{4 k_{2} \eta^{2}}{x_{1}}\left(x_{2} \sin x_{6}-x_{3} \cos x_{6}\right) \\
\dot{b}_{3}=\frac{k_{3}}{x_{1}}\left(x_{4}^{2}+x_{5}^{2}-\tan ^{2} \frac{i_{d}}{2}\right)\left(x_{4}^{2}+x_{5}^{2}+1\right)\left(x_{2} x_{5}-x_{3} x_{4}+x_{5} \cos x_{6}-x_{4} \sin x_{6}\right)
\end{gathered}
$$

Inspection of Eqs. (37)-(39) reveals that subset $1\left(x_{1}=0\right)$ does not belong to the invariant set, therefore convergence toward rectilinear trajectories is ruled out. Instead, both subsets 2 and 3 form the invariant set for the problem at hand.

Actually, convergence toward subset 2 is only theoretical. In fact, the Lyapunov function can be rewritten in terms of orbit elements as 


$$
V=\frac{1}{2}\left[k_{1}\left(p-p_{d}\right)^{2}+k_{2}\left(e^{2}-e_{d}^{2}\right)^{2}+k_{3}\left(\tan ^{2} \frac{i}{2}-\tan ^{2} \frac{i_{d}}{2}\right)^{2}\right]
$$

where $p, e$, and $i$ are the instantaneous semilatus rectum, eccentricity, and inclination. The partial derivative of $V$ with respect to $i$ is

$$
\frac{\partial V}{\partial i}=k_{3} \tan \frac{i}{2}\left(\tan ^{2} \frac{i}{2}+1\right)\left(\tan ^{2} \frac{i}{2}-\tan ^{2} \frac{i_{d}}{2}\right)
$$

It is apparent that $\partial V / \partial i=0$ (i.e. $V$ is stationary) at $i=0$, which is consistent with the fact that subset 2 belongs to the invariant set. However, if $i=i_{\varepsilon}>0$ (with $i_{\varepsilon}$ arbitrarily small), then $\partial V / \partial i<0$, and the reduction of $V$ leads $i$ to increasing up to the desired value $i_{d}$, which is associated with subset 3 , i.e. the target set. This circumstance has the very interesting practical consequence that - from the numerical point of view - the dynamical system of interest enjoys global convergence toward the desired operational conditions, provided that the control law (28) is adopted, while holding the assumptions of either Proposition 1, 2, or 3.

\section{NONLINEAR CONTROL ON VERY LOW EARTH ORBIT}

Some mission scenarios involve microsatellites placed in low Earth orbits, where their main task is the periodic monitoring of specific target areas. Sometimes, several satellites form constellations. In the absence of correction maneuvers, the performance attainable from these microsatellites degrades relatively quickly due to orbit perturbations. In particular, at very low altitudes, that is, $400 \mathrm{~km}$ or less, aerodynamic drag plays a crucial role, and yields orbit decay, i.e. a substantial and progressive altitude reduction. Orbit maintenance has the objective of avoiding these degradation effects and is usually performed employing chemical propulsion.

This study investigates the use of low-thrust electric propulsion for the purpose of accurate orbit maintenance for a microsatellite with initial mass of $30 \mathrm{~kg}$, equipped with two Busek ion thrusters [48]. Their overall propulsive performance is identified by the following parameters:

$$
c=24.124 \mathrm{~km} / \mathrm{sec} \quad \text { and } \quad n_{T}^{(\max )}=1.292 \cdot 10^{-5} \mathrm{~g}_{0} \quad\left(\mathrm{~g}_{0}=9.8 \mathrm{~m} / \mathrm{sec}^{2}\right)
$$

The aerodynamic surface, which is assumed to coincide with the cross section subject to solar radiation $\left(S_{D} \equiv S_{R}\right.$ ), equals $0.785 \mathrm{~m}^{2}$ (and corresponds to that of a sphere with radius of $0.5 \mathrm{~m}$ ).

The desired orbit is circular, with altitude of $400 \mathrm{~km}$ and inclination of $50 \mathrm{deg}$. Hence, due to the definitions of Eq. (1), the desired operational conditions correspond to Eqs. (29) through (31), with $p_{d}=R_{E}+400 \mathrm{~km}, e_{d}=0$, and $i_{d}=50 \mathrm{deg}$. The spacecraft motion is governed by Eqs. (3), (5), and (17). The initial RAAN and argument of latitude are both set to 0. Errors at orbit injection are also considered, and the initial conditions for the 7 state components are

$x_{1, i}=6778.136 \mathrm{~km} \quad x_{2, i}=4.426 \cdot 10^{-3} \quad x_{3, i}=0 \quad x_{4, i}=0.488 \quad x_{5, i}=0 \quad x_{6, i}=0 \quad x_{7, i}=1$

These initial conditions correspond to perigee and apogee altitudes of $370 \mathrm{~km}$ and $430 \mathrm{~km}$, respectively, whereas the inclination equals $52 \mathrm{deg}$.

Orbit acquisition and maintenance using low-thrust ion propulsion employs the feedback control law defined in Eq. (28). With this regard, the preceding section supplies three sets of sufficient conditions (stated in Propositions 1 through 3) that ensure asymptotic stability, i.e. convergence toward the desired operational conditions (29)-(31). In particular, the hypotheses of Propo- 
sitions 1 and 2 can be checked only a posteriori (and often do not hold at all times). Thus, as a first step, the conditions stated in Proposition 3 are being analyzed. At the initial time $t_{0}$, the spacecraft has minimum thrust acceleration, because $x_{7}\left(t_{0}\right)=1$. The inequality $u_{T}^{(\max )} / x_{7}\left(t_{0}\right)=u_{T}^{(\max )}>\left|\boldsymbol{a}_{P}\right|$ is thus checked, making reference to the operational orbit of interest. Using the spacecraft data (i.e. propulsion, mass, and surface), the maximal magnitude of the perturbing acceleration turns out to exceed the minimum available thrust acceleration $u_{T}^{(\max )}$, due to the major contributions of some Earth gravitational harmonics, especially $J_{2}$. Yet, on average the $J_{2}$ perturbation does not change the orbit semimajor axis, eccentricity, and inclination. Among the remaining harmonics, only the $J_{2,2}$ harmonic yields an acceleration with maximum magnitude that exceeds $u_{T}^{(\max )}$. Hence, Proposition 3 cannot guarantee convergence toward the target set. The next step is in considering Proposition 2, which relates the sign of $\dot{V}$ to the term $\boldsymbol{b}^{T} \boldsymbol{a}_{P}$. All the Earth harmonics yield oscillating accelerations in an orbital period, as well as some of the remaining perturbations (solar radiation pressure and third body gravitational pull). As a result, $\boldsymbol{b}^{T} \boldsymbol{a}_{P}$ assumes both positive and negative values. However, $\dot{V}$ depends on two terms (cf. Eq. (24)): (i) the first, negative term, and (ii) the second term, whose sign is time-varying and is given by that of $\boldsymbol{b}^{T} \boldsymbol{a}_{P}$. Due to this, one can conjecture that the inequality $\dot{V}<0$ is met on average, albeit it is not fulfilled instantaneously. If this conjecture is correct, then $V$ assumes an oscillating time behavior, with negative average time derivative. On the other hand, it is worth remarking that $x_{7}$, which represents the mass ratio, is a decreasing function of time, therefore the inequality $u_{T}^{(\max )} / x_{7}>\left|\boldsymbol{a}_{P}\right|$ can be satisfied as the spacecraft mass reduces even if it was not met at the initial time.

In conclusion, the feedback control law (28) has the clear potentiality of driving the system toward the invariant set, more specifically toward the target set, in light of all the considerations drawn in the present and the preceding sections. Effectiveness of the feedback control law (28) is being tested numerically in the next subsections.

\subsection{Numerical results without eclipse effect}

Ion thrusters require onboard electrical power in order to operate. In some cases, this can be provided only when the space vehicle is illuminated. However, in this subsection, ion thrusters are assumed to be able to operate regardless of the spacecraft lighting conditions.

In practical scenarios, specific tolerances may be allowed on the desired final conditions, in order to avoid excessive propellant expenditure. Specifically, if the perigee and apogee radii are constrained to prescribed intervals, then Eqs. (29) and (30) are treated as fulfilled, by setting $k_{1}=k_{2}=0$, i.e.

$$
\frac{x_{1}}{1+\sqrt{x_{2}^{2}+x_{3}^{2}}} \geq R_{E}+380 \mathrm{~km} \quad \text { and } \quad \frac{x_{1}}{1-\sqrt{x_{2}^{2}+x_{3}^{2}}} \leq R_{E}+420 \mathrm{~km} \quad \Rightarrow \quad k_{1}=k_{2}=0
$$

Similarly, if the orbit inclination is contained in a specified interval, then $k_{3}=0$, i.e.

$$
49.5 \mathrm{deg} \leq 2 \arctan \left\{\sqrt{x_{4}^{2}+x_{5}^{2}}\right\} \leq 50.5 \mathrm{deg} \quad \Rightarrow \quad k_{3}=0
$$


The numerical simulations are performed using canonical units. The distance unit (DU) equals the Earth radius, whereas the time unit (TU) is such that $\mu_{E}=1 \mathrm{DU}^{3} / \mathrm{TU}^{2}$. Moreover, the following weighting coefficient are used, after extensive trial-and-attempt tuning: $k_{1}=1, k_{2}=1$, and $k_{3}=100$. Orbit propagations are performed for a duration of 5 years and the initial reference epoch is set to 1 June 2020 at 12 am GMT. The overall propellant expenditure equals $1.625 \mathrm{~kg}$. From inspection of Figs. 1 and 2 it is apparent that the perigee and apogee radii are driven within the specified tolerances (denoted with horizontal lines), as well as the orbit inclination. Figure 2 depicts also the mass ratio time history, pointing out that two phases exist: (a) orbit acquisition, where the thrust magnitude is maximum, and (b) orbit maintenance. Figure 3 portrays the time evolution of $V$, which exhibits of short-period oscillations (in the inset), while the average behavior of $V$ is time-decreasing, as conjectured in the previous subsection. Finally, Fig. 4 illustrates the thrust components. Inspection of this figure reveals that during phase (b) the thrust direction is horizontal (no radial and normal thrust component is in fact applied).
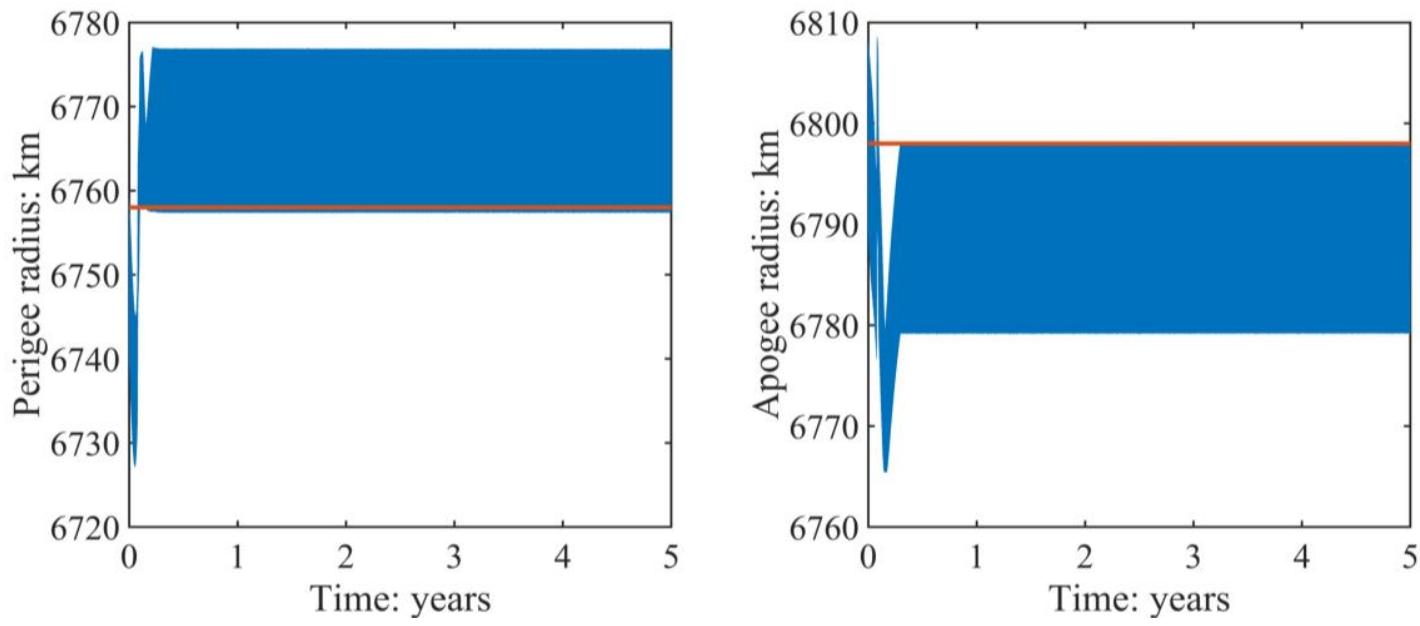

Figure 1. LEO satellite (without eclipse effect): time histories of the perigee and apogee radii
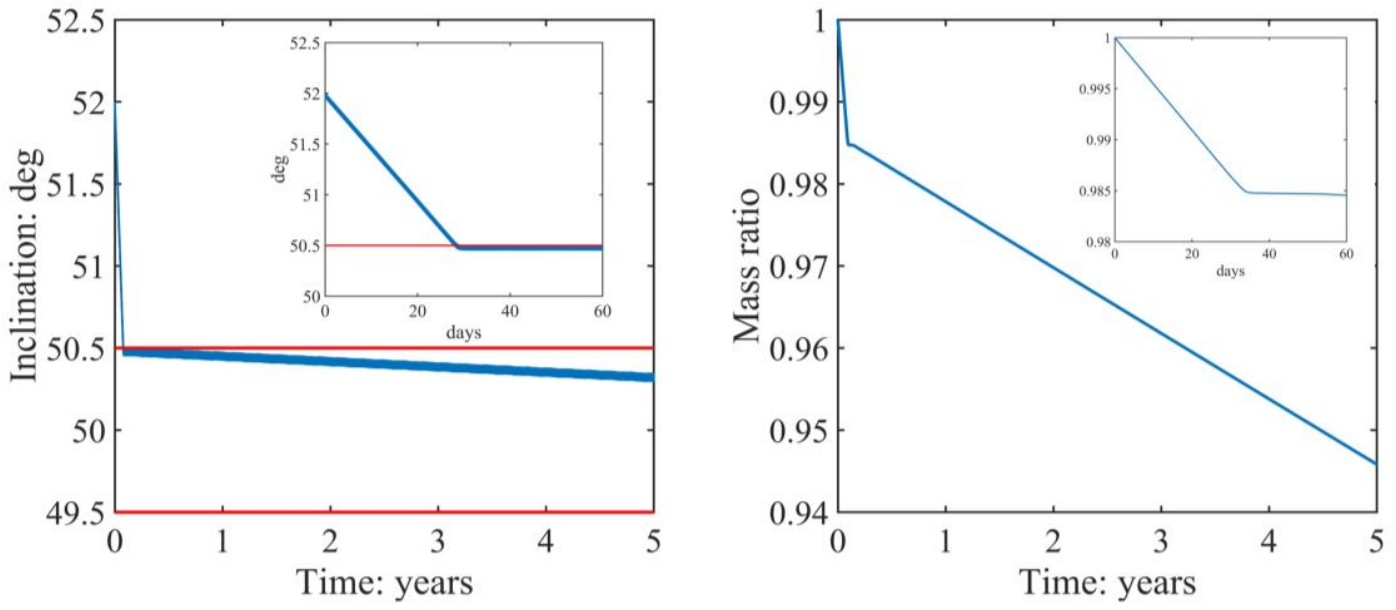

Figure 2. LEO satellite (without eclipse effect): time histories of inclination (with zoom in the inset) and mass ratio 

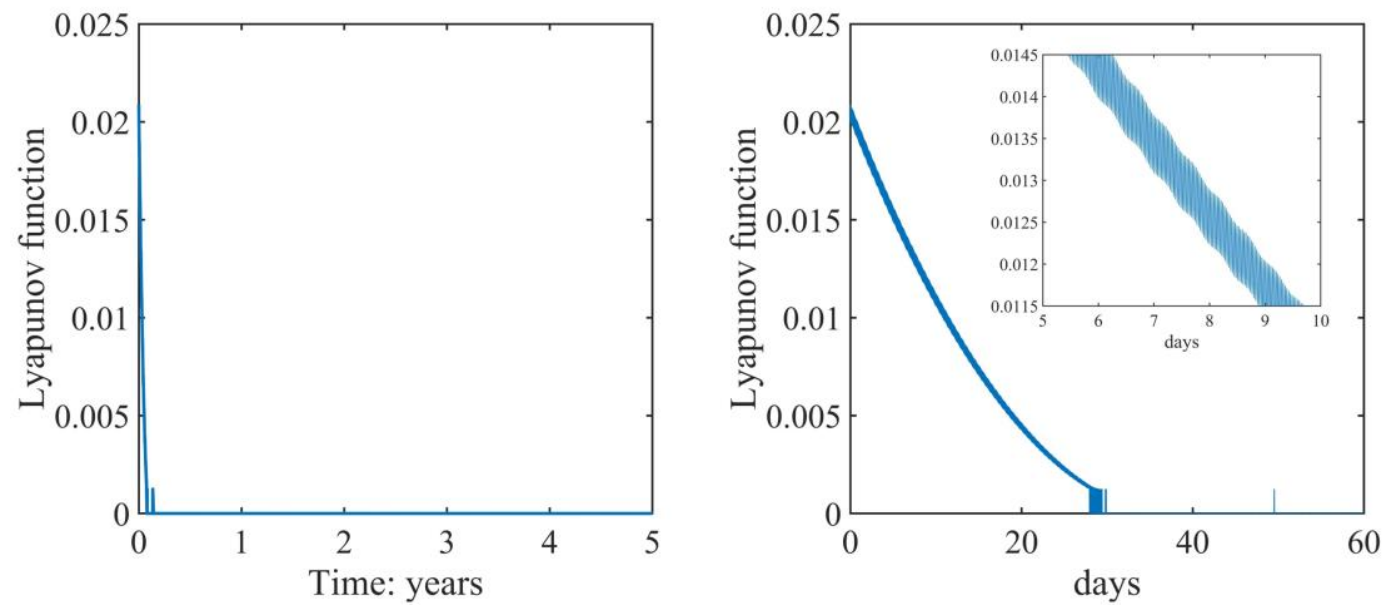

Figure 3. LEO satellite (without eclipse effect): time history of the Lyapunov function (with zoom in the inset of the right figure)
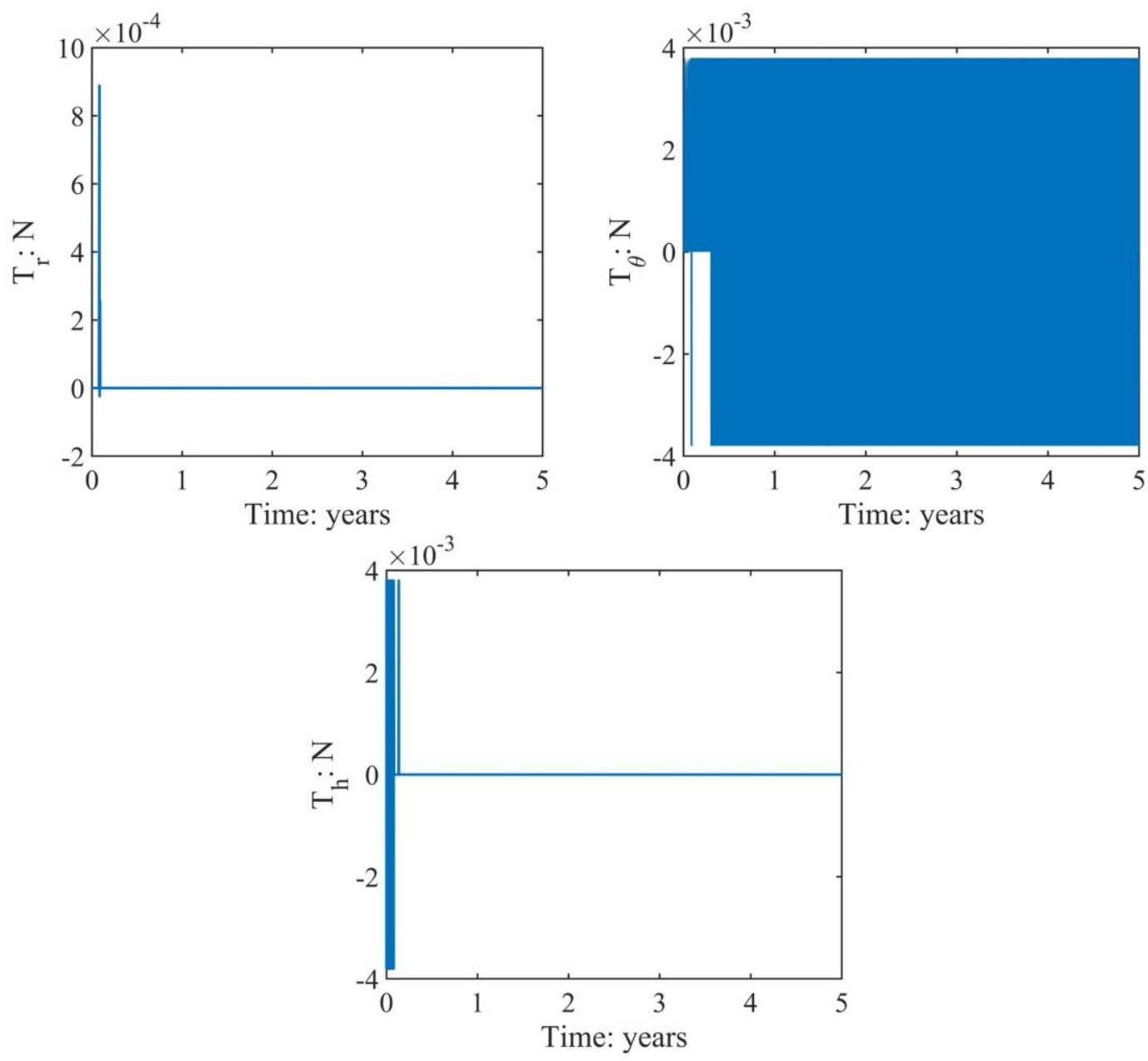

Figure 4. LEO satellite (without eclipse effect): time histories of the thrust components 


\subsection{Numerical results with eclipse effect}

This subsection includes the eclipse effect on the available electric power. This means that ion propulsion is considered unavailable when the microsatellite is not illuminated. The eclipse conditions are identified using the shadow function $v$, introduced in Section 3.4.

The tolerances (44)-(45) are assumed also in this case. The canonical units are those employed in the previous subsection, whereas the weighting coefficients, selected after trial-and-attempt tuning, are $k_{1}=1, k_{2}=10^{7}$, and $k_{3}=10^{2}$.

Orbit propagations are performed for a duration of 5 years and the initial reference epoch is set again to 1 June 2020 at 12 am GMT. The overall propellant expenditure equals $2.451 \mathrm{~kg}$. From inspection of Figs. 5 and 6 it is apparent that the perigee and apogee radii are driven within the specified tolerances, as well as the orbit inclination, with some exceptions limited to the time intervals where ion propulsion is off due to eclipse. Figure 6 portrays also the mass time history, which has faster decrease in the early phases, due to correction of orbit injection errors. Subsequently, the ion propulsion is activated and turned off repeatedly and this is apparent from inspection of Fig. 8, which illustrates the thrust components. Unlike the previous simulations in the absence of the eclipse effect, the thrust is applied also in the radial direction after orbit acquisition. Figure 7 portrays the time evolution of $V$, which exhibits again short-period oscillations (in the inset), while the average behavior of $V$ is time-decreasing.
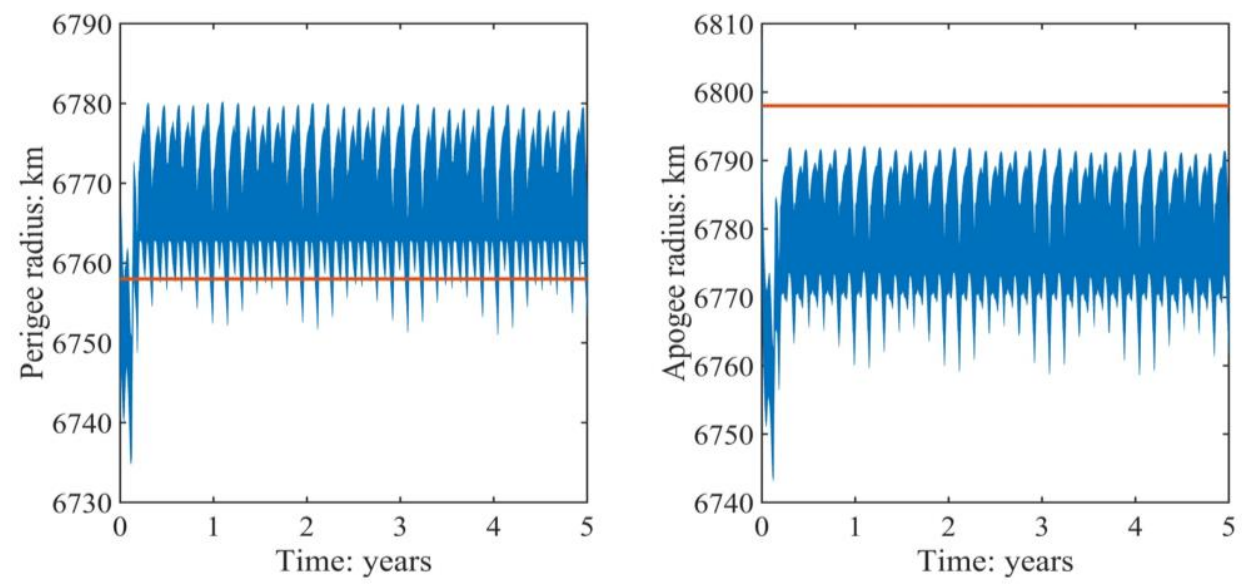

Figure 5. LEO satellite (with eclipse effect): time histories of the perigee and apogee radii
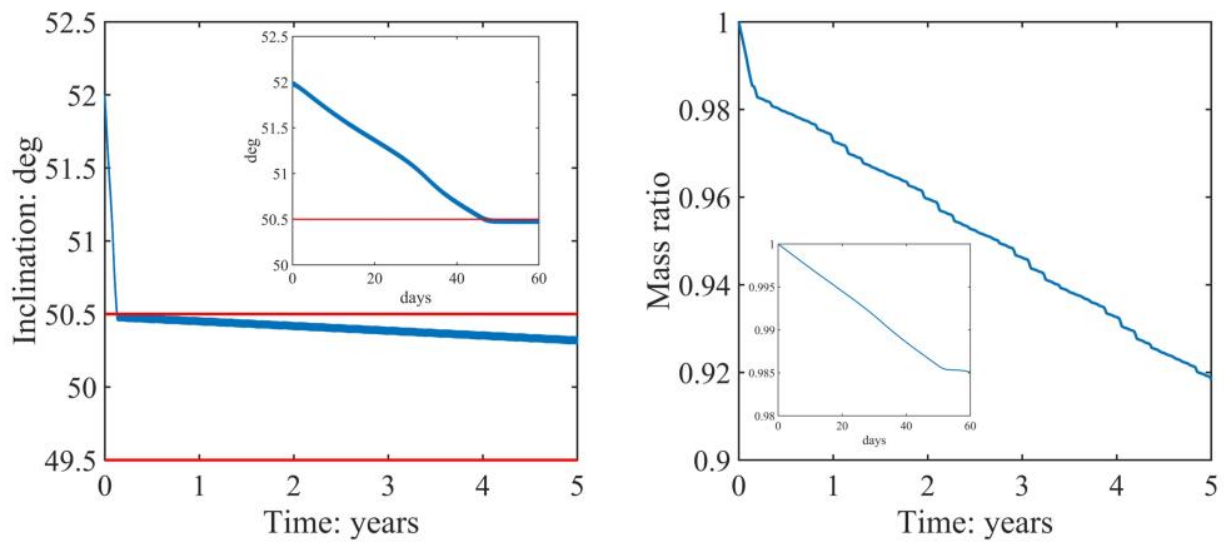

Figure 6. LEO satellite (with eclipse effect): time histories of inclination and mass (with zoom in the inset) 

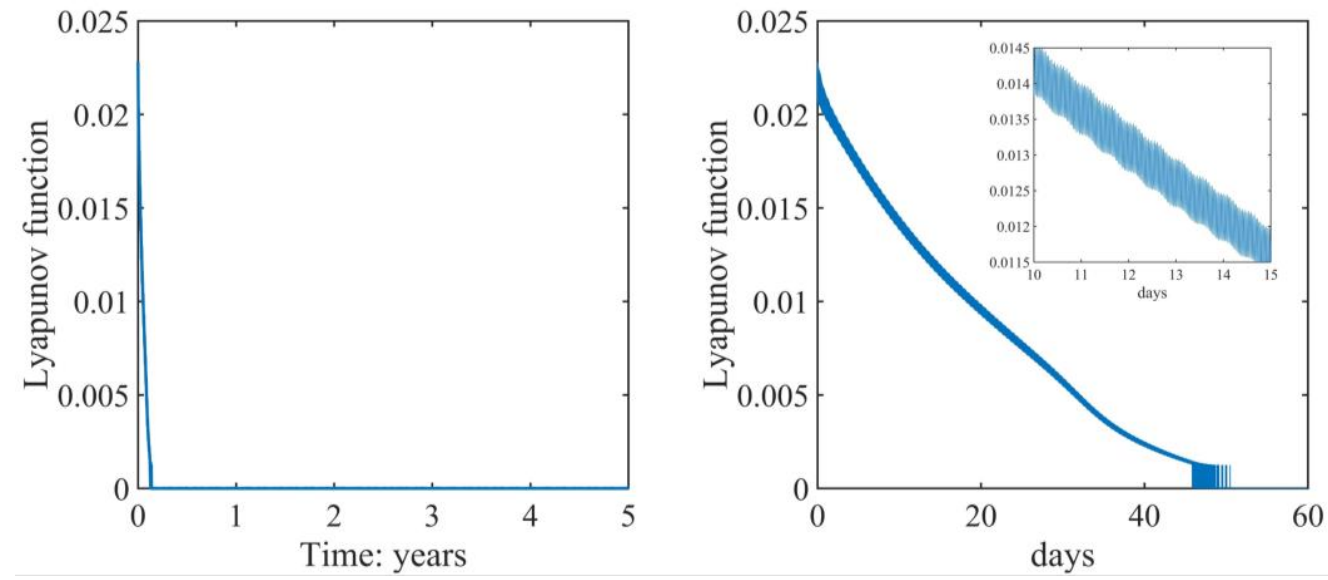

Figure 7. LEO satellite (with eclipse effect): time history of the Lyapunov function (with zoom in the inset of the right figure)
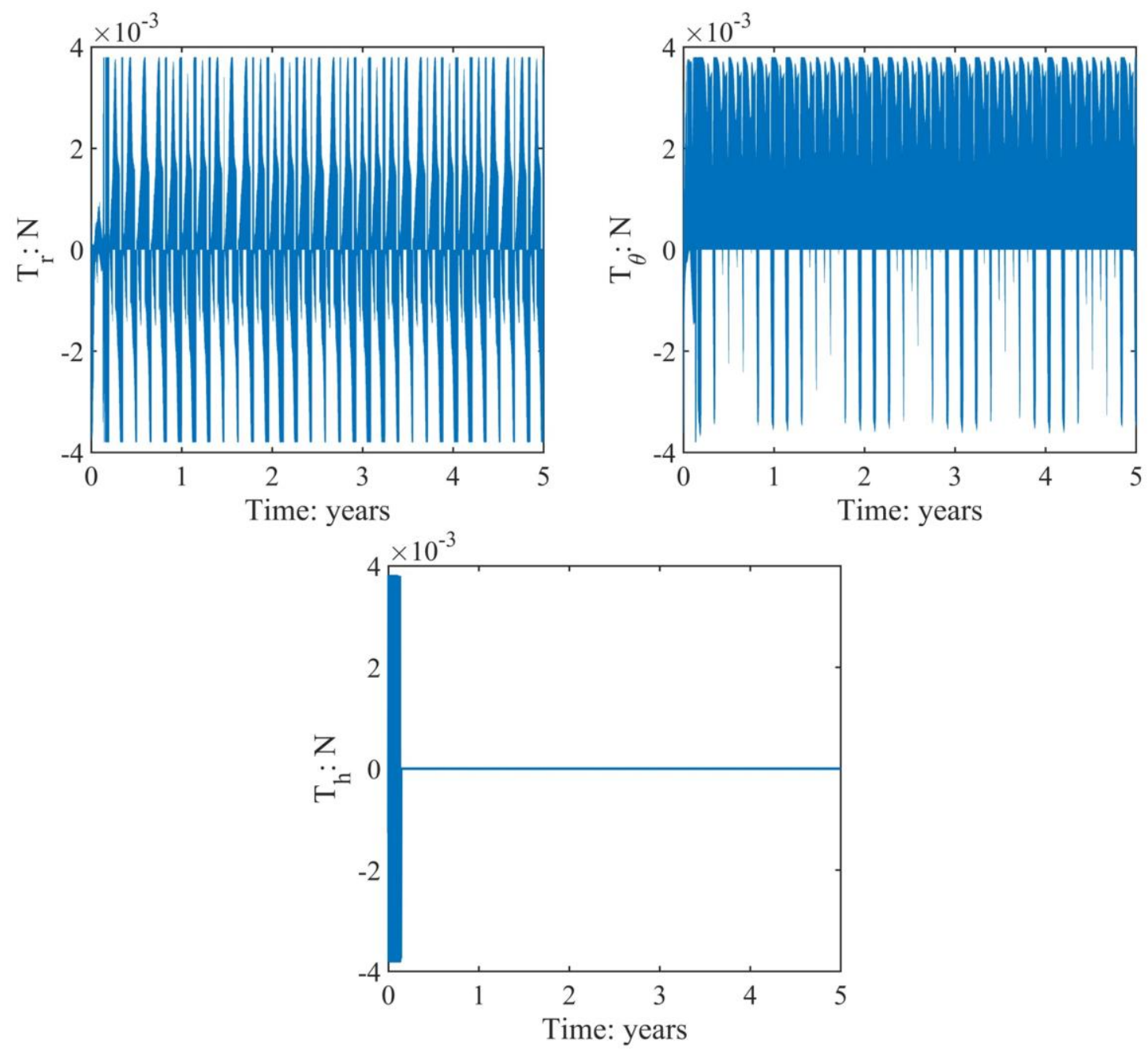

Figure 8. LEO satellite (with eclipse effect): time histories of the thrust components 


\section{NONLINEAR CONTROL ON MEDIUM-ALTITUDE EARTH ORBIT}

Alternative mission scenarios involve satellites placed in medium-altitude Earth orbits, where their main task is often in providing navigation services. In the absence of correction maneuvers, the performance attainable from these satellites degrades again due to orbit perturbations. Orbit maintenance has the objective of avoiding these degradation effects.

In this section, low-thrust propulsion is used again for accurate orbit injection and maintenance. For the purpose of comparing the performance of nonlinear control, the same spacecraft considered in the preceding section is assumed. Ion propulsion is considered again, with the parameters specified in Eq. (42). At the altitudes that are being considered, drag is absent. Instead, solar radiation pressure plays a role, and the related spacecraft cross section equals $0.785 \mathrm{~m}^{2}$.

The desired orbit is elliptic, with perigee and apogee altitudes equal to $23206 \mathrm{~km}$ and 23237 $\mathrm{km}$, respectively, whereas the inclination is $56.6 \mathrm{deg}$. These are the typical orbit elements of the satellites that form the Galileo constellation and are chosen for illustrative purposes as representative values for the class of medium-altitude Earth satellites. Hence, the desired operational conditions correspond again to Eqs. (29) through (31), with $p_{d}=29599.628 \mathrm{~km}, e_{d}=5.097 \cdot 10^{-4}$, and $i_{d}=56.6 \mathrm{deg}$. The spacecraft motion is governed by Eqs. (3), (5), and (17). The initial RAAN, argument of perigee, and true anomaly are all set to 0 . Errors at orbit injection are considered, and the initial conditions for the 7 state components are

$$
x_{1, i}=29084.355 \mathrm{~km} \quad x_{2, i}=0.1 \quad x_{3, i}=0 \quad x_{4, i}=0.466 \quad x_{5, i}=0 \quad x_{6, i}=0 \quad x_{7, i}=1
$$

Orbit acquisition and maintenance using low-thrust ion propulsion employs the feedback control law defined in Eq. (28). With this regard, Section 4 supplies three sets of sufficient conditions (stated in Propositions 1 through 3 ) that ensure asymptotic stability, i.e. convergence toward the desired operational conditions (29)-(31). Similarly to the previous case, the hypotheses of Propositions 1 and 2 can be checked only a posteriori and are frequently violated, thus only the conditions stated in Proposition 3 are being analyzed.

At the initial time $t_{0}$, the spacecraft has minimum thrust acceleration, because $x_{7}\left(t_{0}\right)=1$. The inequality $u_{T}^{(\max )} / x_{7}\left(t_{0}\right)=u_{T}^{(\max )}>\left|\boldsymbol{a}_{P}\right|$ is thus checked, referring to the operational orbit of interest. Using the spacecraft data (i.e. propulsion, mass, and surface), the minimum available thrust ac-

celeration $u_{T}^{(\max )}$ turns out to exceed the maximal magnitude of the perturbing acceleration, unlike what occurs for spacecraft orbiting the Earth at low altitudes. As a consequence, the feedback control law is expected to drive the spacecraft toward the desired operational conditions, in light of the global stability properties established in Section 4. Effectiveness of the feedback control law (28) is being tested numerically in the next subsections.

\subsection{Numerical results without eclipse effect}

Ion thrusters require onboard electrical power in order to operate. In some cases, this can be provided only when the space vehicle is illuminated. However, in this subsection, ion thrusters are assumed to be able to operate regardless of the spacecraft lighting conditions.

Similarly to the case of low-thrust orbits, specific tolerances may exist on the periapse and apoapse radii. However, for medium-altitude orbits, Proposition 3, together with the subsequent theoretical developments, ensures global asymptotic convergence. In this subsection, convergence is verified numerically. To do this, no tolerance on the desired orbit elements is introduced, and the operational conditions defined previously are pursued. 
The numerical simulations are performed using canonical units. The distance unit (DU) equals the Earth radius, whereas the time unit (TU) is such that $\mu_{E}=1 \mathrm{DU}^{3} / \mathrm{TU}^{2}$. Moreover, the following weighting coefficient are used, after extensive trial-and-attempt tuning: $k_{1}=1, k_{2}=10^{4}$, and $k_{3}=1$. Orbit propagations are performed for a duration of 5 years and the initial reference epoch is set to 1 June 2020 at 12 am GMT. The overall propellant expenditure equals $7.234 \mathrm{~kg}$. From inspection of Figs. 9 and 10 it is apparent that the semimajor axis, eccentricity, and inclination converge to the desired operational values. Figure 10 depicts also the mass ratio time history, pointing out that two phases exist: (a) orbit acquisition, where the thrust magnitude is maximum, and (b) orbit maintenance. Figure 11 portrays the evolution of $V$, which is decreasing in time. This is consistent with the expected global asymptotic convergence toward the operational conditions. Finally, Fig. 12 illustrates the thrust components and magnitude. Inspection of this figure reveals that the thrust components have similar amplitudes, both in phase (a) and in phase (b).
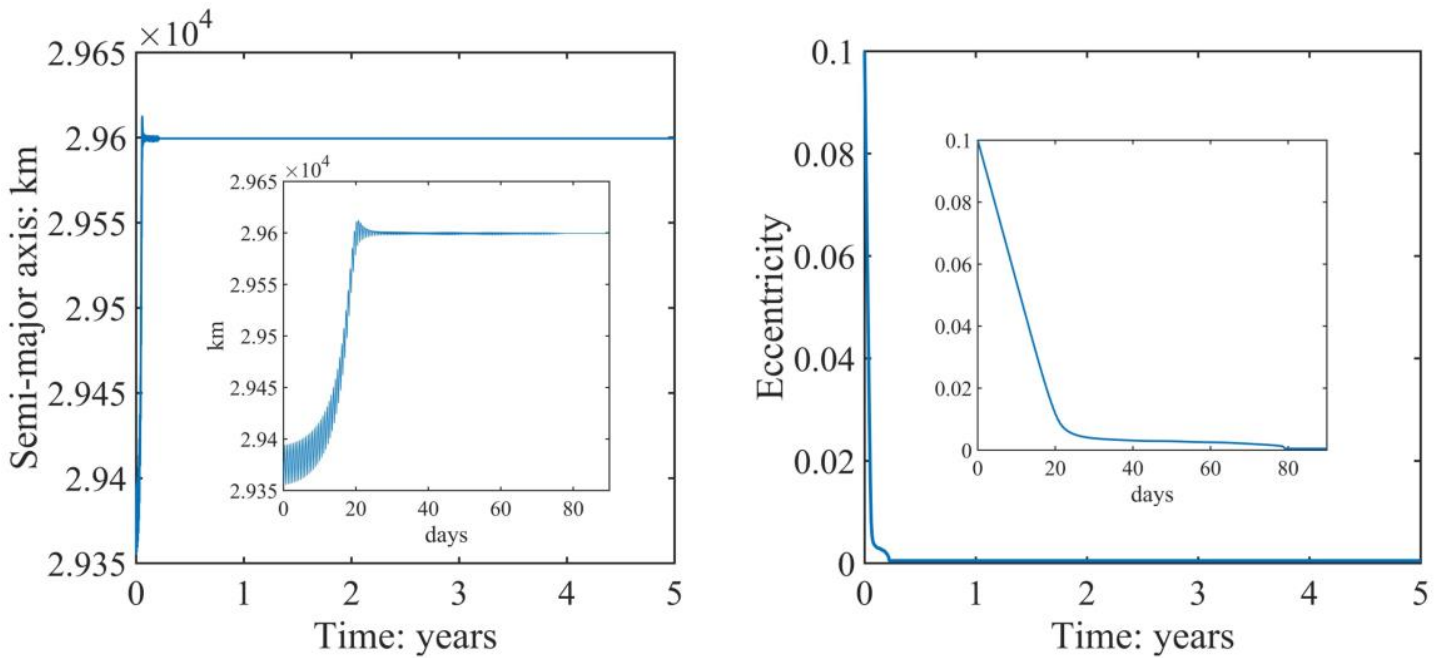

Figure 9. MEO satellite (without eclipse effect): time histories of the semimajor axis and eccentricity (with zoom in the insets)
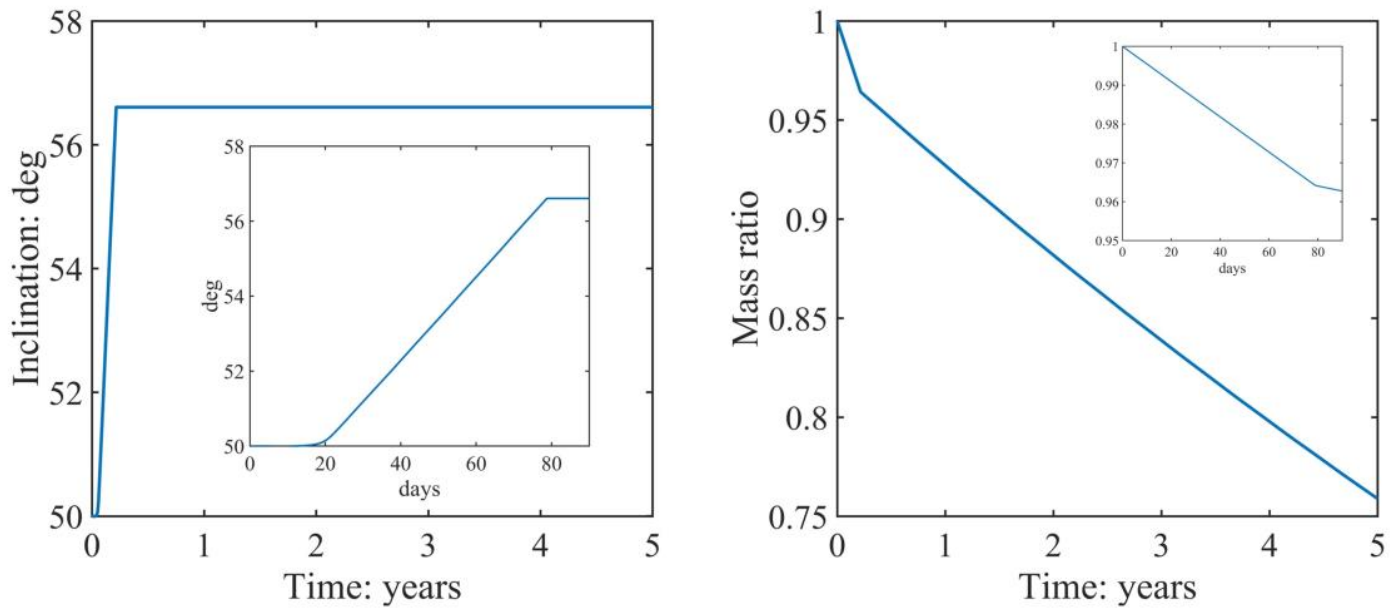

Figure 10. MEO satellite (without eclipse effect): time histories of inclination and mass (with zoom in the insets) 

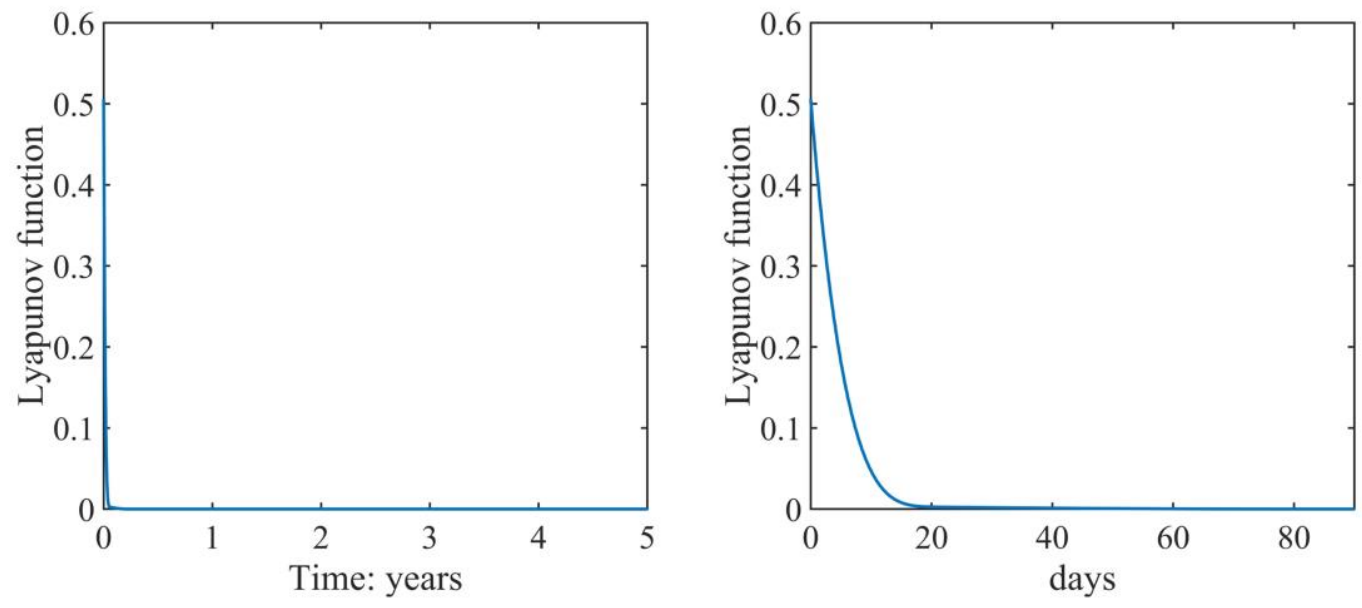

Figure 11. MEO satellite (without eclipse effect): time history of the Lyapunov function
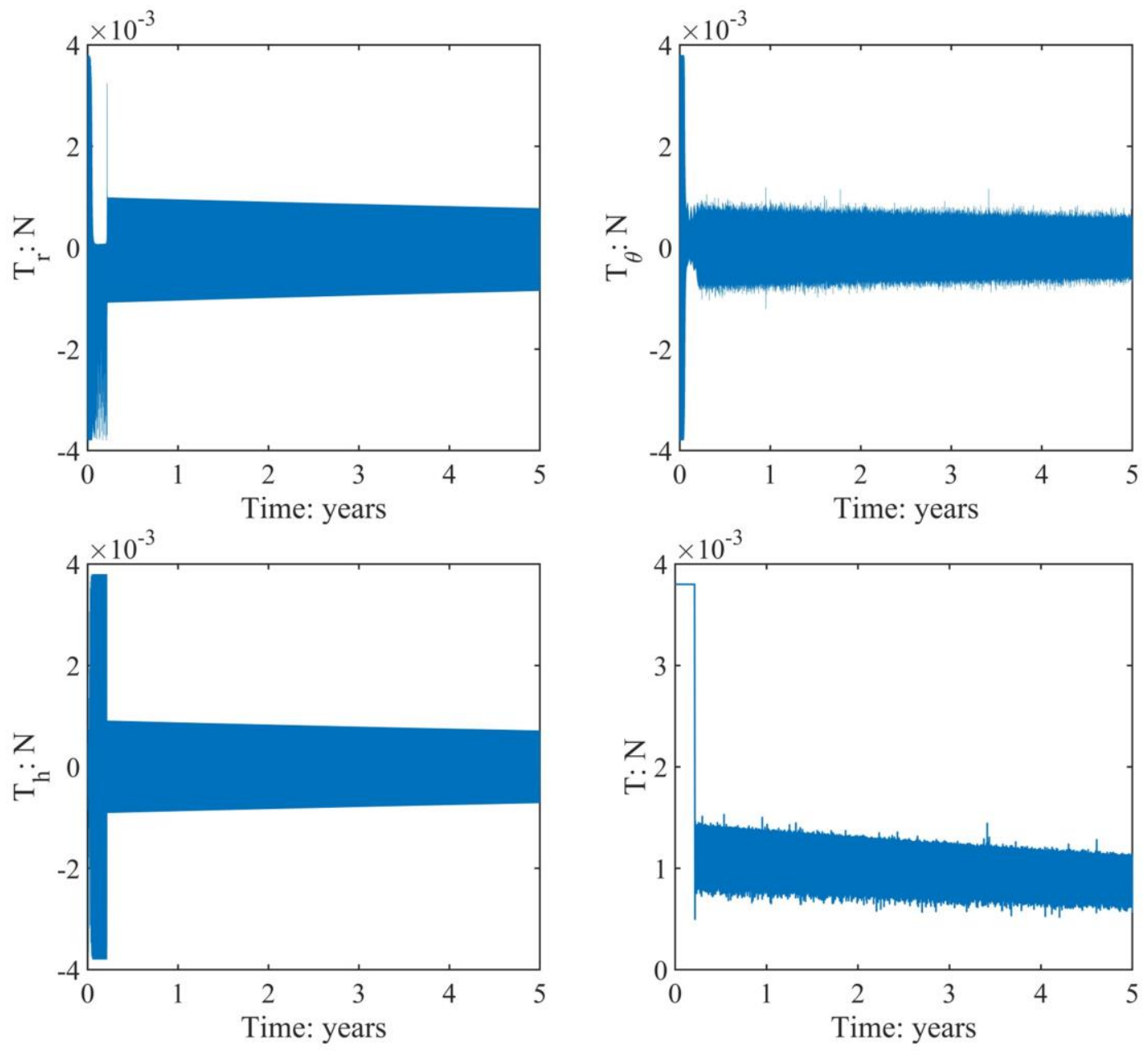

Figure 12. MEO satellite (without eclipse effect): time histories of the thrust components and magnitude 


\subsection{Numerical results with eclipse effect}

This subsection includes the eclipse effect on the available electric power. This means that ion propulsion is considered unavailable when the microsatellite is not illuminated. The eclipse conditions are identified using the shadow function $v$, introduced in Section 3.4.

No tolerance is assumed also in this case. The canonical units and the weighting coefficients $\left\{k_{1}, k_{2}, k_{3}\right\}$ are those employed in the previous subsection.

Orbit propagations are performed for a duration of 5 years and the initial reference epoch is set again to 1 June 2020 at 12 am GMT. The overall propellant expenditure equals $6.949 \mathrm{~kg}$. From inspection of Figs. 13 and 14 it is apparent that the semimajor axis, eccentricity, and inclination converge to the desired values. However, the semimajor axis exhibits some oscillations due to unavailability of thrust during the eclipse intervals. Figure 14 portrays also the mass time history, which has faster decrease in the early phases, due to correction of orbit injection errors.
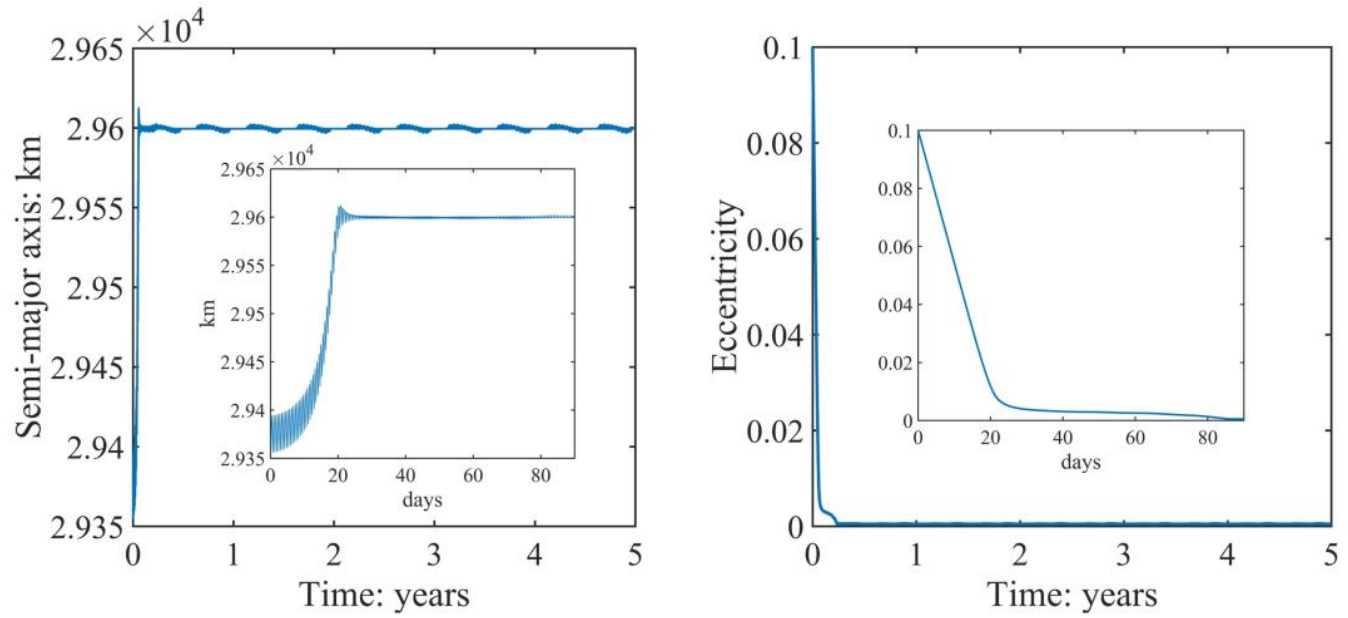

Figure 13. MEO satellite (with eclipse effect): time histories of the semimajor axis and eccentricity (with zoom in the insets)
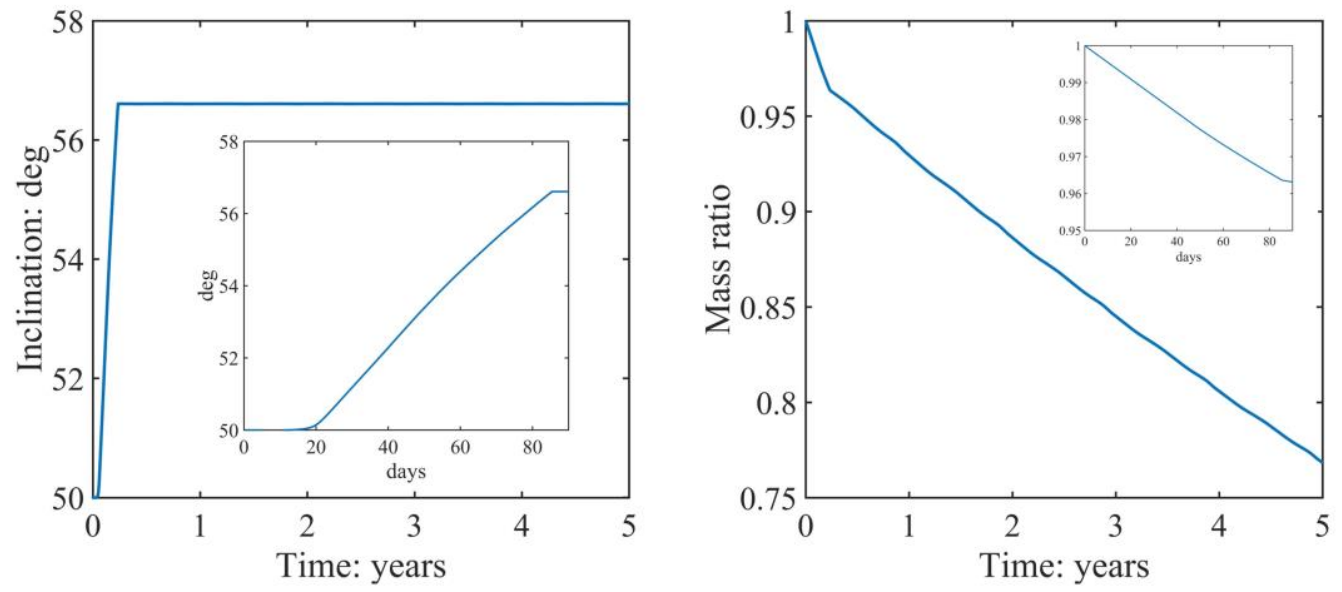

Figure 14. MEO satellite (with eclipse effect): time histories of inclination and mass (with zoom in the insets) 

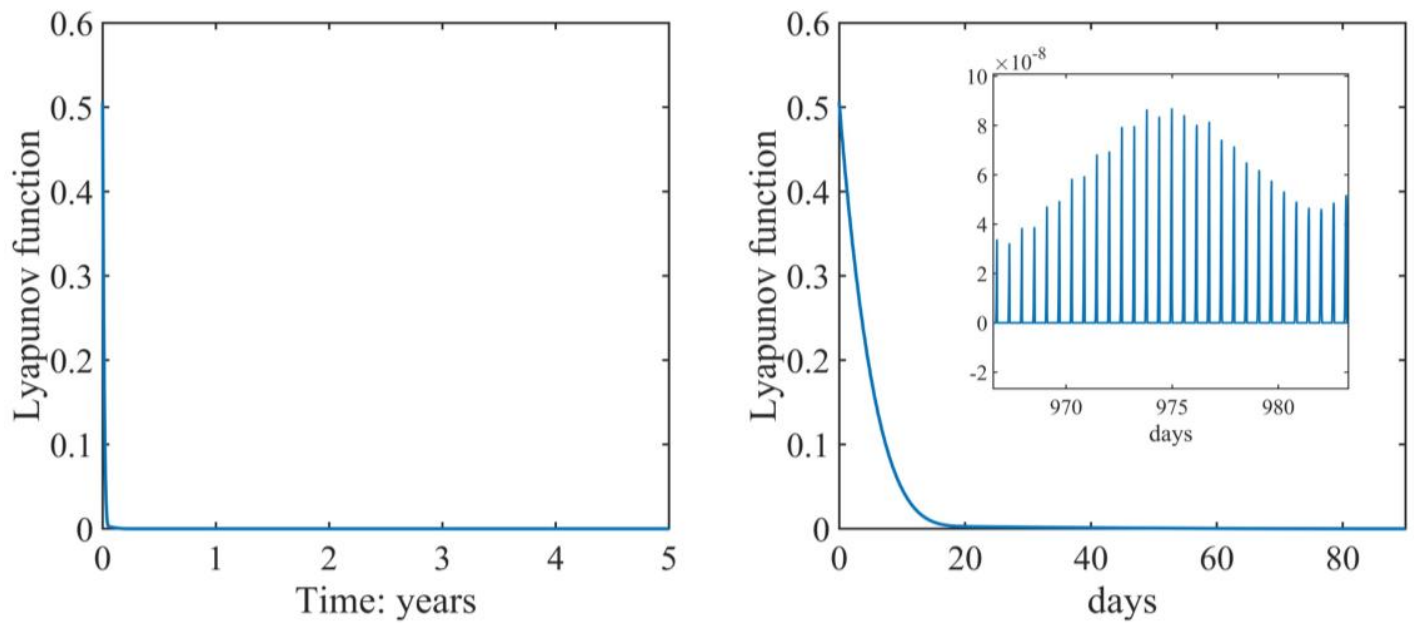

Figure 15. MEO satellite (with eclipse effect): time history of the Lyapunov function
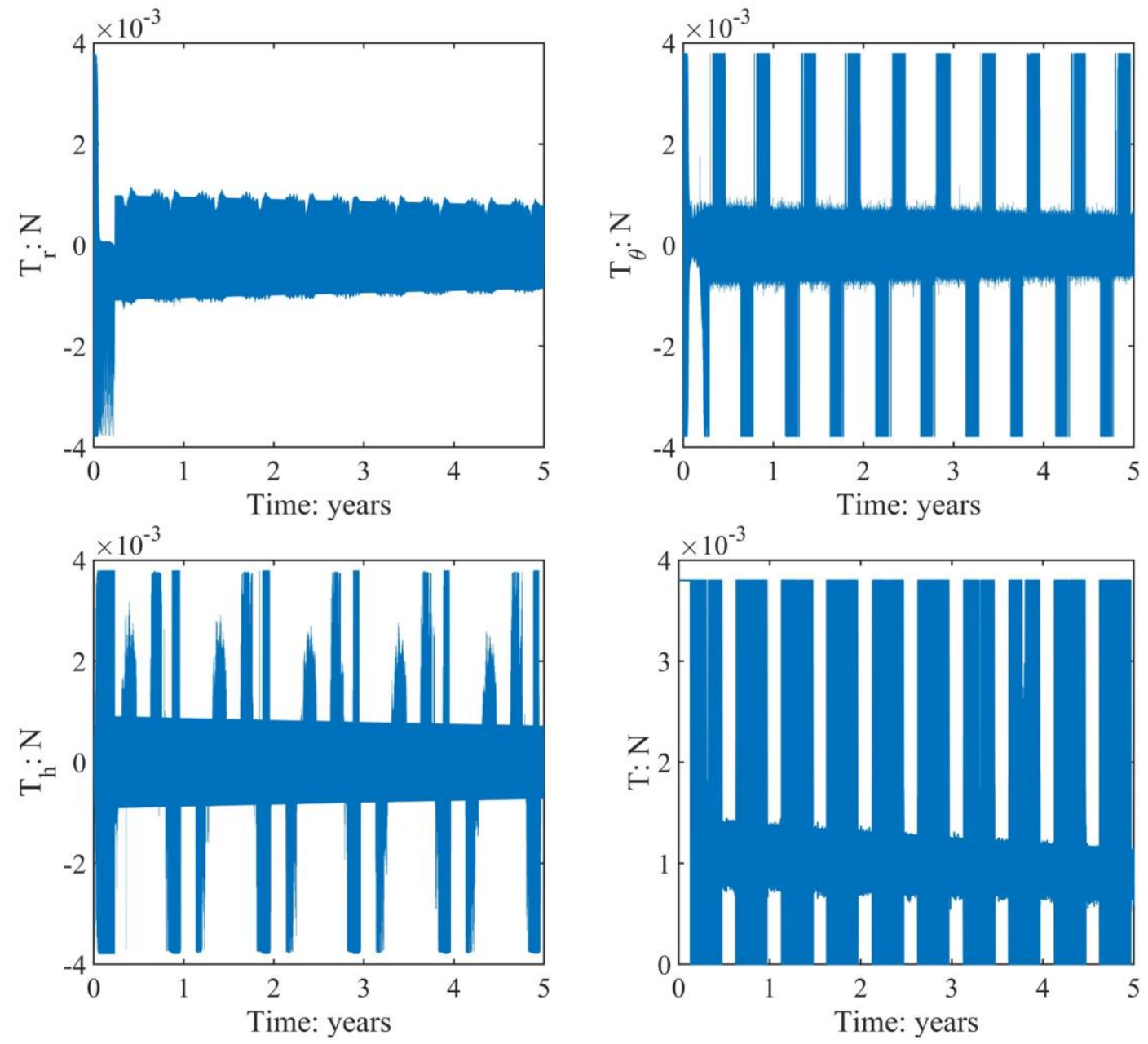

Figure 16. MEO satellite (with eclipse effect): time histories of the thrust components and magnitude 
Subsequently, ion propulsion is activated and turned off repeatedly and this is apparent from inspection of Fig. 15, which illustrates the time history of the Lyapunov function. In fact, the modest-amplitude spikes portrayed in the inset correspond to the eclipse intervals. Unlike the previous simulations in the absence of the eclipse effect, the maximum thrust is applied repeatedly, even after orbit acquisition (cf. Fig. 16), which takes a relatively short time (i.e., about 20 days, cf. insets of Fig. 13). In Fig. 16 it is apparent that all the thrust components have similar amplitudes.

\section{CONCLUDING REMARKS}

This research addresses the definition, analysis, and numerical testing of a real-time feedback orbit control strategy tailored to compensating orbit perturbations and errors at orbit injection for spacecraft placed in Earth orbits. The space vehicle of interest is assumed to be equipped with a throttleable, steerable ion propulsion system, with an upper bound on the thrust magnitude.

Lyapunov stability theory, in conjunction with the LaSalle's invariance principle, supply the theoretical foundations for the definition of a feedback control law capable of driving the dynamical system at hand toward the desired operational conditions. Unlike several former contributions published in the scientific literature, this study proposes a saturated control law for orbit maneuvering, in the presence of perturbing accelerations inherent to the space environment. Moreover, the operational conditions, which identify the target set, are expressed in a rather general form. They are proven to correspond to a portion of the invariant set of the controlled system. Asymptotic convergence toward the target set is thus demonstrated, and it is global in all the dynamical contexts of practical interest, if some sufficient conditions, stated in three distinct Propositions, are met.

Two mission scenarios, corresponding to two different operational orbits, are investigated: (a) circular, very low Earth orbit and (b) near-circular, medium-altitude Earth orbit. All the relevant orbit perturbations are included in the dynamical model used for numerical propagations. Due to the modest thrust level associated with the ion propulsion system, the sufficient conditions for stability are violated in mission scenario (a). In fact, Earth gravitational harmonics are responsible of oscillating perturbing accelerations, whose maximal magnitude in low Earth orbits exceeds the available thrust acceleration magnitude. Nevertheless, for scenario (a) the sufficient conditions for stability hold on average. As a result, convergence and stabilization in the proximity of the desired operational conditions is demonstrated, albeit short-period oscillations cannot be avoided and represent the ultimate, non-compensated effect of Earth gravitational harmonics. Suitable tolerances on the operational conditions allow substantial propellant savings, because propulsion is switched on only when the flight conditions are beyond some specified bounds. As a further effort to model real scenarios with enhanced fidelity, satellite eclipsing is considered. In this case, the numerical simulations demonstrate that the tolerances are occasionally violated in some limited time intervals (where the ion propulsion is unavailable). In mission scenario (b), the expected asymptotic convergence toward the operational conditions - with no tolerance - is demonstrated numerically. Satellite eclipsing yields modest oscillations of the semimajor axis, during the intervals when the ion propulsion is unavailable. All the numerical simulations refer to a duration of 5 years and demonstrate that for both orbits (a) and (b) the propellant consumption is very limited, thus making the nonlinear control technique at hand particularly suitable and appealing for precise orbit injection and maintenance.

\section{ACKNOWLEDGEMENTS}

The authors wish to thank Simone Proietti for some useful discussions on several numerical aspects of the nonlinear control technique described in this work. 


\section{REFERENCES}

[1] P. Gurfil and P. K. Seidelmann. Celestial Mechanics and Astrodynamics: Theory and Practice, Springer, Berlin Heidelberg, Germany, 2016, pp. 369-410

[2] T. N. Edelbaum. "Some Extensions of the Hohmann Transfer Maneuver." ARS Journal, Vol. 29, 1959, pp. 864865

[3] L. Ting. "Optimum Orbital Transfer by Several Impulses." Astronautica Acta, Vol. 6/Fasc. 5, 1960, pp. 256-265

[4] D. F. Lawden. Optimal Trajectories for Space Navigation, Butterworths, London, 1963, pp. 79-110

[5] R. B. Barrar. "An Analytic Proof that the Hohmann-type Transfer is the True Minimum Two-Impulse Transfer." Astronautica Acta, Vol. IX/Fasc. 1, 1963, pp. 1-11

[6] J.-P. Marec. Optimal Space Trajectories, Elsevier, Amsterdam, 1979, pp. 21-27

[7] R. H. Battin. An Introduction to the Mathematics and Methods of Astrodynamics, AIAA Education Series, AIAA, New York, 1987, pp. 448-450, 490-494, 529-530

[8] J. I. Palmore. "An Elementary Proof of the Optimality of Hohmann Transfers." Journal of Guidance, Control, and Dynamics, Vol. 7, No. 5, 1984, pp. 629-630

[9] J. E. Prussing. "Simple Proof of the Global Optimality of the Hohmann Transfer." Journal of Guidance, Control, and Dynamics, Vol. 15, No. 4, 1992, pp. 1037-1038

[10] G. A. Hazelrigg. "Globally Optimal Impulsive Transfers via Green's Theorem." Journal of Guidance, Control, and Dynamics, Vol. 7, No. 4, 1983, pp. 462-470

[11] M. Pontani. "Simple Method to Determine Globally Optimal Orbital Transfers." Journal of Guidance, Control, and Dynamics, Vol. 32, No. 3, 2009, pp. 899-914

[12] G. P. Sutton and O. Biblarz. Rocket Propulsion Elements, John Wiley and Sons, New York, NY, 2001, pp. 660710

[13] M. D. Rayman, P. A. Chadbourne, J. S. Culwell, S. N. Williams. "Mission Design for Deep Space 1: A Lowthrust Technology Validation Mission." Acta Astronautica. Vol. 45, No. 4-9, 1999, pp. 381-388

[14] P. Rathsman, J. Kugelberg, P. Bodin, G. D. Racca, B. Foing, L. Stagnaro "SMART-1: Development and lessons learnt." Acta Astronautica, Vol. 57, No. 2-8, 2005, pp. 455-468

[15] T. N. Edelbaum. "The use of high- and low-thrust propulsion in combination for space missions." Journal of the Astronautical Sciences, Vol. 9, 1962, pp. 58-59

[16] A. E. Petropoulos, "Simple Control Laws for Low-Thrust Orbit Transfers." AIAA/AAS Astrodynamics Specialist Conference, Big Sky, MN, 2003

[17] A. E. Petropoulos, "Low-Thrust Orbit Transfers Using Candidate Lyapunov Functions with a Mechanism for Coasting." AIAA/AAS Astrodynamics Specialist Conference and Exhibit, Providence, RI, 2004

[18] J. T. Betts, "Very low-thrust trajectory optimization using a direct SQP method." Journal of Computational and Applied Mathematics, Vol. 120, 2000, pp. 27-40

[19] J. T. Betts and S. O. Erb, "Optimal Low Thrust Trajectories to the Moon." SIAM Journal of Applied Dynamical Systems, Vol. 2, No. 2, 2003, pp. 144-170

[20] J. T. Betts, "Optimal low thrust orbit transfers with eclipsing." Optimal Control Applications and Methods, Vol. 36,2015 , pp. $218-240$

[21] I. M. Ross, Q. Gong, and P. Sekhavat, "Low-Thrust, High-Accuracy Trajectory Optimization." Journal of Guidance, Control, and Dynamics, Vol. 30, No. 4, 2007, pp. 921-933

[22] J. A. Kéchichian, "Optimal Low-Thrust Rendezvous Using Equinoctial Orbit Elements." Acta Astronautica, Vol. 38, No. 1, 1996, pp. 1-14

[23] J. A. Kéchichian, "Optimal Low-Thrust Transfer in General Circular Orbit Using Analytic Averaging of the System Dynamics." The Journal of the Astronautical Sciences, Vol. 57, 2009, pp. 369-392

[24] J. A. Kéchichian, "Low-Thrust Inclination Control in Presence of Earth Shadow." Journal of Spacecraft and ceRockets, Vol. 35, 1998, pp. 1015-1017 
[25] J. A. Kéchichian, "Mathematics of attitude-constrained optimal low-thrust orbit transfer." Aerotecnica Missili \& Spazio, the Journal of Aerospace Science, Technology and Systems, Vol. 96, No. 4, 2017, pp. 180-194

[26] M. Pontani and B. A. Conway. "Minimum-Fuel Finite-Thrust Relative Orbit Maneuvers via Indirect Heuristic Method." Journal of Guidance, Control, and Dynamics, Vol. 38, No. 5, 2015, pp. 913-924

[27] M. Pontani and B. A. Conway. "Optimal Low-Thrust Orbital Maneuvers via Indirect Swarming Method." Journal of Optimization Theory and Applications, Vol. 162, No. 1, 2014, pp. 272-292

[28] J. T. Betts. "Survey of Numerical Methods for Trajectory Optimization." Journal of Guidance, Control, and Dynamics, Vol. 21, No. 2, 1998, pp. 193-207

[29] A. Rao. "A Survey of Numerical Methods for Optimal Control." Advances in the Astronautical Sciences, Vol. 135, No. 1, 2009, pp. 497-528

[30] B. A. Conway. "A Survey of Methods Available for the Numerical Optimization of Continuous Dynamical Systems." Journal of Optimization Theory and Applications, Vol. 152, 2012, pp. 271-306

[31] C. A. Kluever. "Simple Guidance Scheme for Low-Thrust Orbit Tranfers." Journal of Guidance, Control, and Dynamics, Vol. 21, No. 6, 1998, pp. 1015-1017

[32] P. Gurfil. "Nonlinear feedback control of low-thrust orbital transfer in a central gravitational field." Acta Astronautica, Vol. 60, 2007, pp. 631-648

[33] H. Schaub and K. T. Alfriend "Hybrid Cartesian and orbit elements feedback law for formation flying spacecraft." Journal of Guidance, Control, and Dynamics, Vol. 25, No. 2, 2002, pp. 339-345, 387-393

[34] M. Pontani, G. Cecchetti, and P. Teofilatto. "Variable-time-domain neighboring optimal guidance applied to space trajectories." Acta Astronautica, Vol. 115, 2015, pp. 102-120

[35] M. Pontani and F. Celani. "Variable-time-domain neighboring optimal guidance and attitude control of low-thrust lunar orbit transfers." Acta Astronautica, Vol. 175, 2020, pp. 616-626

[36] H. K. Khalil. Nonlinear Systems, Prentice Hall, Upper Saddle River, NJ, 2002, pp. 111-194

[37] E. D. Sontag. Mathematical Control Theory, Springer, New York, NY, 1998, pp. 183-260

[38] S. Sastry. Nonlinear Systems. Analysis, Stability, and Control, Springer, New York, NY, 1999, pp. 182-234

[39] J-J. E. Slotine and W. Li. Applied Nonlinear Control, Prentice Hall, Englewood Cliffs, NJ, 1991, pp. 40-99

[40] J. E. Prussing and B. A. Conway. Orbital Mechanics, Oxford University Press, New York, NY, 2013, pp. 46-54

[41] H. Schaub and J. L. Junkins. Analytical Mechanics of Space Systems, AIAA Education Series, Reston, VA, 2003, pp. $519-525$

[42] R. A. Broucke and P. J. Cefola, "On the Equinoctial Orbit Elements." Celestial Mechanics, Vol. 5, 1972, pp. 303310

[43] N. K. Pavlis, S. A. Holmes, S. C. Kenyon, and J. K. Factor. "An Earth Gravitational Model to Degree 2160 : EGM2008." General Assembly of the European Geosciences Union, Vienna, Austria, 2008

[44] S. Giorgi. "Una Formulazione Caratteristica del Metodo di Encke in Vista dell'Applicazione Numerica." Scuola di Ingegneria Aerospaziale, Rome, Italy, 1964

[45] H. D. Curtis. Orbital Mechanics for Engineering Students, Elsevier, Kidlington, Oxford, U.K., 2014, pp. 695-715

[46] D. A. Vallado. Fundamentals of Astrodynamics and Applications, Microcosm Press, El Segundo, CA, 2004, pp. 524-542

[47] V. Jurdjevic and J. P. Quinn. "Controllability and Stability." Journal of Differential Equations, Vol. 28, 1978, pp. 381-389

[48] D. Williams. "Propulsion Solutions for CubeSats and Applications." CubeSat Developers Workshop, Logan, UT, 2012 